\title{
Charging effects and Andreev reflection in a double-junction circuit: A model approach combining rate equations and Green's functions
}

\author{
Ursula Schröter* and Elke Scheer \\ Fachbereich Physik, Universität Konstanz, Universitätsstraße 10, 78457 Konstanz, Germany \\ (Received 20 April 2006; revised manuscript received 7 August 2006; published 5 December 2006)
}

\begin{abstract}
We present a qualitative model for current transport in the superconducting state through a series of two quantum-point contacts with a mesoscopic island between them. A Green's functions technique is merged with a rate-equation method in order to account for phase as well as charging effects. Multiple Andreev reflections are included in a nonperturbative manner and therefore our Ansatz despite some underlying assumptions is in principle not restricted to the low or high transmission regime. We find that in our system, multiple Andreev reflection is not totally suppressed by Coulomb blockade, but that the step pattern of current-voltage characteristics is determined by an interplay of multiple thresholds.
\end{abstract}

\section{INTRODUCTION}

Electronic transport through two constrictions in series has been well investigated in two limiting cases. For two tunnel junctions with low transmission transport is described by orthodox theory (OT), and Coulomb blockade (CB) is the dominating effect. ${ }^{1}$ The other well-treated kind of configuration is the central island consisting of a sufficiently short diffusive wire, ${ }^{2}$ a quantum dot, ${ }^{3,4}$ or a molecule ${ }^{5}$ with few discrete energy levels. A theoretical approach to handle the interaction between the leads and the island for widely open transmission channels has been made by bosonization of the Hamiltonian, ${ }^{6-8}$ predicting $\mathrm{CB}$ effects for low temperature.

We here propose a model for quantum point contacts (QPCs) with channels of arbitrary transmission. The island between them is supposed to be metallic, same as the leads. It has to be small enough to present a capacitance sensitive to single-charge effects, however, large enough to exhibit a bulklike continuous density of states. This is realizable with metallic areas of micrometer diameter and a few tenths of nanometers height. By quantum point contacts $^{9}$ we mean junctions across which interaction is based on phasesensitive wave-function overlaps and characterizable in terms of transport channels. ${ }^{10}$ A good example for quantum point contacts are single-atom contacts which can be arranged with mechanically controllable break junctions. ${ }^{9}$ Single-atom contacts are known to exhibit few transport channels, ${ }^{11,12}$ the number of which depends on the valence of the metal used and which can have arbitrary transmissions. Our theoretical method, based on a Green's functions ${ }^{13,14}$ as well as a rate-equation technique ${ }^{15}$ consists in combining the following features in one model for the double-junction case: We include coherent multiple reflections and multiple Andreev reflections (MARs) as well as interference between different-order processes in each of the junctions. However, we assume that coherence is not maintained in transport across the island between the two contacts. Nevertheless the model accounts for simultaneousness and mutual influence of transport processes in both junctions via the shifting island potential due to charging. The main questions behind our investigations are whether for the two-junction series MARs actually require multiple excess charges ${ }^{16}$ to accumulate on the island and whether MARs get suppressed by CB. Our model aims at demonstrating the principle feasibility of a nonperturbative Ansatz and simulating qualitative behavior. Exact mapping to experiments is not expected. Quantitative correctness of absolute current values, step heights in the current-voltage curves or the shape of steps and plateaus is not necessarily claimed. The important aspect is the prediction of voltage thresholds for the onsets of charge transport processes requiring coherent interaction across each junction. The model produces correct results in the limit of perfect transmissions and is able to mimic preliminary experimental observations for small transmissions. ${ }^{17}$

Section II presents the main steps of our calculation procedure; details can be found in the Appendixes. In Sec. III we show some examples and interpret our results in an intuitive energy-level scheme, which will be supported by a deeper analysis in Sec. IV. We conclude with a summary and an outlook in Sec. V.

\section{MODEL}

The circuit considered is shown in Fig. 1. An island is linked to a left and a right lead by quantum point contacts (QPCs) each characterized by a capacitance and an ensemble of transport channels. The island is further coupled capacitively to a gate electrode which allows one to shift its potential [Fig. 1(a)]. Each channel in the junctions [Fig. 1(b)] has a transmission probability $\theta$ between zero and one. The equivalent resistance of each channel would be $R=\frac{h}{2 e^{2}} \frac{1}{\theta}$. The transmission probability $\theta$ is converted to a transmission amplitude $t$ by $\theta=\frac{4 t^{2}}{\left(1+t^{2}\right)^{2}} \cdot{ }^{13}$ This renormalization of the transmission stems from summing up all multiple normal reflections in a single-channel contact, characterized by $t$, in the normal state. The transmission amplitude is assumed to be independent of the energy of the charge-carrying particle.

All three sites (the left and right reservoirs $L$ and $R$ and the island $I$ ) are taken to be bulk superconductors with the same $\Delta$ [Fig. 1(c)]. For contacts with dimensions smaller than the coherence length the junctions can be modeled as nonsuperconducting regions of zero length with a deltalike barrier. Their properties are, however, much more easily de- 
(a)

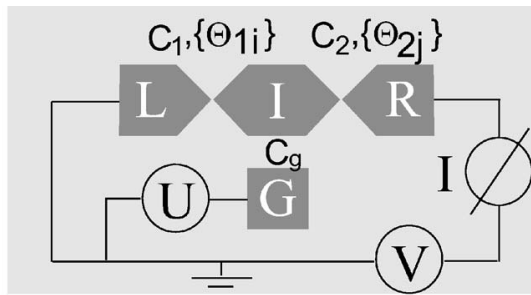

(b)

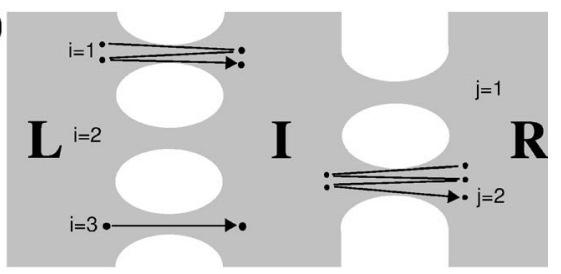

(c)

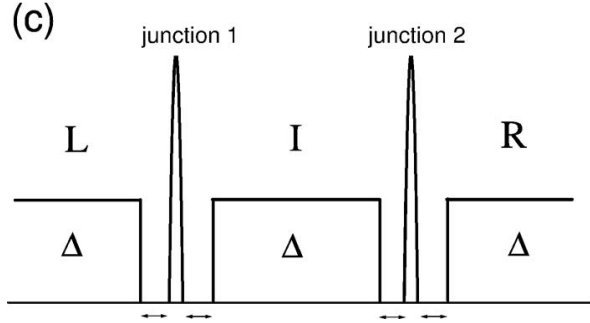

FIG. 1. (a) Scheme of the setup considered: An island between two junctions towards a left and a right lead, characterized by capacitances $C_{1}$ and $C_{2}$ as well as channels with transmissions $\theta_{1 i}$ and $\theta_{2 j}$, respectively, as well as a gate with capacitance $C_{g} . i=1,2,3$ and $j=1,2$ in (b) is only an example for the number of channels. $V$ and $U$ are the applied transport and gate voltage, $I$ is the current to deduce. (b) Charge transport: Coherent multiple (Andreev) reflections are only allowed within each channel, however, processes may be simultaneous in different channels and in both junctions. (c) Scheme of the model system showing the spatial dependence of the gap parameter, which is the same in $L, I$, and $R$. The junctions are represented as barriers. The length of the $\leftrightarrow$ regions is taken as zero (Ref. 18).

fined by the sets of transmission coefficients $\left\{\theta_{1 i}\right\}$ and $\left\{\theta_{2 j}\right\}$. References 18 and 13 show on the single junction that the region-with-barrier and the transport-channel picture are equivalent. Both Ansätze produce exactly the same currentvoltage curves that perfectly fit the experiments. ${ }^{11,12}$ In our model all three sites $L, R$, and $I$ are associated a BCS singleparticle density of states (see Fig. 8) with Fermi level at the center of a $2 \Delta$-wide gap, and with filled electron or empty hole states below and empty electron or filled hole states above the gap. We work in the single-particle picture. No temperature effects are included yet; the Fermi function for occupation is always taken at zero temperature. We also restrict ourselves to dissipative transport; that is, no supercurrents due to resonant Cooper-pair tunneling are considered in our model. This might neglect additional current peaks that would be very narrow in the voltage, however. ${ }^{19,20}$

Each of the three sites $L, I$, and $R$ has its own order parameter, the phase of which only depends on the voltage of the site. By order parameter we mean the pseudo-wavefunction $\Psi(\vec{r})=\sqrt{n_{S}} e^{i \varphi(\vec{r})}$ accorded to all superconducting charge carriers in common after London theory. The link to the single (quasi)particle picture will be contained in the Green's functions. The reservoir potentials are fixed at zero on the left and $V$ on the right. The gate voltage $U$ induces a charge $C_{g} U$ on the island. Any further charge on the island has to be quantized, and $n$ additional negative charges move the potential to $\Phi=\left(V C_{2}+U C_{g}-n e\right) / C_{\text {tot }}$ with $C_{\text {tot }}=C_{1}+C_{2}$ $+C_{g}$ the total capacitance of the island towards the rest of the circuit. ${ }^{21}$ Let $A=\left(V C_{2}+U C_{g}\right) / C_{\text {tot }}$ denote the island potential with respect to the left lead for no extra charges on it $(n$ $=0)$ and $C=\left[(U-V) C_{g}-V C_{1}\right] / C_{\text {tot }}$ the island potential measured against the right lead in this case. Then in general, the island potential can be written as $\Phi=A-n B$ (or $A$ replaced by $C$ with the right lead for reference instead of the left) with $B=e / C_{\text {tot }}$, closely related to the so-called charging energy $E_{c}=e^{2} / 2 C_{\text {tot }}$.

It might seem a problem that we take both the number of charges on the island and the phase of the order parameter as well defined quantities. Our model relies on the assumption that a small number of excess electrons or holes does neither alter the density of states nor the occupation of states according to the zero-temperature Fermi distribution, because the excess charges only represent a very small fraction of the electron or hole gas on the metal island. Only the Fermienergy level, always marking the middle of the gap, is shifted. This is a good approximation for a large enough island. The number of charges is only used to evaluate the classical electrostatic potential. In the quantum-mechanical sense only the phase is determined, so no contradiction to the uncertainty relation between conjugate variables is constructed. Regarding a junction, it is, however, reasonable to view charges as localized on either side of it, as even a perfectly open channel with $\theta=1$ means a $13 k \Omega$ barrier between a lead and the island. Thus the basis of states we adopt corresponds to what is usually known as "phase states," but in this work we nevertheless prefer to call them "charge states" referring to the respective Fermi-potential levels. There will be interference between these states in the form of renormalized transmission rates, and at the same time they represent a classical set of states that occur with certain probabilities.

The Keldysh Green's functions formalism, describing operators in the interaction picture, is adapted to using the noninteracting system, that is, the uncoupled sites here, as a basis. We do not need eigenstates of a full Hamiltonian, ${ }^{22}$ although the coupling across the contacts need not be small. The island can take on all the different states corresponding to every possible number $n$ of excess charges [Fig. 2(a)]. To fully describe the state of the system, however, also the basis states for the leads have to contain the information about the actual charge on the island, which will be introduced as upper indices. This is equivalent to the bookkeeping on how many charges have passed a junction in orthodox theory models. States from our basis in $L$ and $I$ or $R$ and $I$ can get coupled by single-particle hoppings if their $n$ differ by 1 [Fig. 2(b)]. Nevertheless, for the leads other than for the island, all $n$ states are at the same potential [Fig. 2(c)]. As here we do not maintain coherence in transport across the island, there is a Hamiltonian for the left lead-island system 


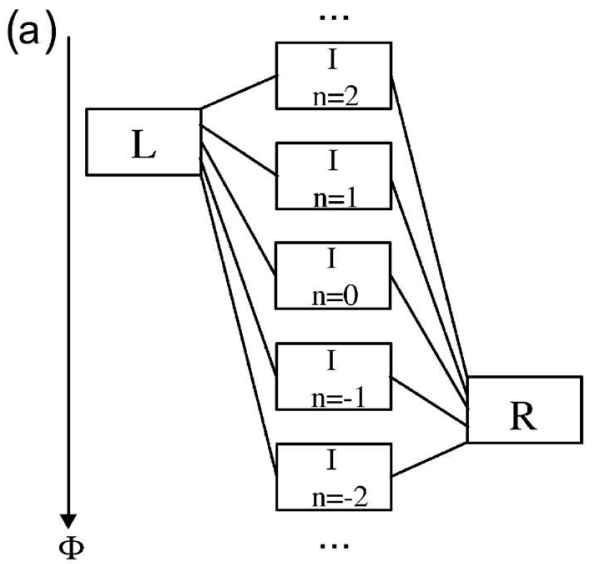

(b)

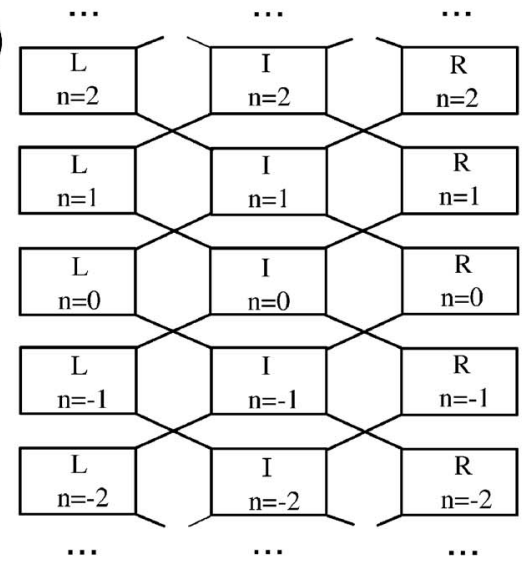

(c)

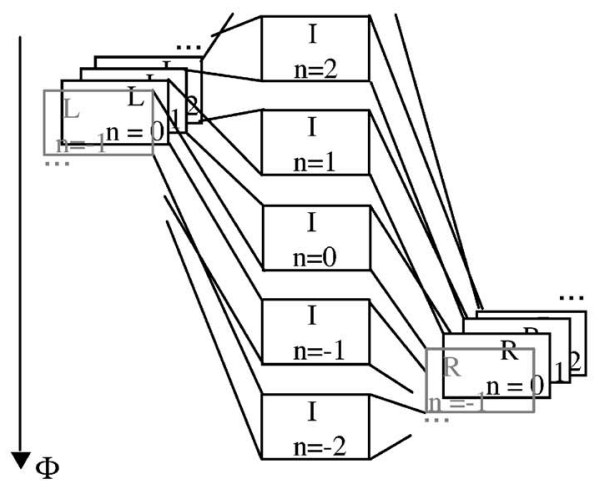

FIG. 2. (a) Sites with island resolved into charge states $n$. (b) Extending the basis in the leads by the $n$ parameter which has, however, (c) no influence on the potential.

$$
\begin{aligned}
\hat{H}_{(L I)}= & \hat{H}_{L}+\hat{H}_{I}+\sum_{n}\left(\sigma_{L I}^{n n-1} c_{L, n}^{\dagger} c_{I, n-1}+\sigma_{L I}^{n n+1} c_{L, n}^{\dagger} c_{I, n+1}\right. \\
& \left.+\sigma_{I L}^{n n-1} c_{I, n}^{\dagger} c_{L, n-1}+\sigma_{I L}^{n n+1} c_{I, n}^{\dagger} c_{L, n+1}\right)
\end{aligned}
$$

and an analogous $\hat{H}_{(R I)}$ for the right lead-island system. The time-independent uncoupled basis ensures that this uses the same states for the island, enabling matching through a rate equation later on. $\hat{H}_{L}$ and $\hat{H}_{I}$ in Eq. (2.1) are understood also to sum over all $n$, and the spin degree of freedom, which is of no interest here, is suppressed in the notation. With the charge-number indices $n, n-1$, and $n+1$, addition energies or chemical potentials are already contained in the creation and annihilation operators $c^{\dagger}$ and $c$ and the coupling $\sigma$, respectively.

Reference 13 derived for a single junction (one channel) the renormalized hopping function $T$ (advanced or retarded). We briefly summarize those results which we use as a starting point here. Sites left and right are denoted with small letters in the following in order not to confuse the single with the double junction. $T$ is got from the implicit Dyson equation

$$
T\left(\tau, \tau^{\prime}\right)=\sigma(\tau) \delta\left(\tau-\tau^{\prime}\right)+\int d \tau_{1} \sigma(\tau) g\left(\tau-\tau_{1}\right) T\left(\tau_{1}, \tau^{\prime}\right)
$$

or written

$$
T=\sigma+\sigma g T
$$

for short and illustrated in Fig. 3(a); $\tau, \tau^{\prime}$, and $\tau_{1}$ are (arbitrary) time arguments. $g$ is the analytically known bulksuperconductor Green's function for the uncoupled sites left and right, and $\sigma$ describes single hoppings. All quantities in Eqs. (2.2) and (2.3) are $2 \times 2$ matrices in electron-hole space

$$
\left(\begin{array}{cc}
T_{e e} & T_{e h} \\
T_{h e} & T_{h h}
\end{array}\right)=\left(\begin{array}{cc}
\sigma_{e} & 0 \\
0 & \sigma_{h}
\end{array}\right)+\left(\begin{array}{cc}
\sigma_{e} & 0 \\
0 & \sigma_{h}
\end{array}\right)\left(\begin{array}{ll}
g_{e e} & g_{e h} \\
g_{h e} & g_{h h}
\end{array}\right)\left(\begin{array}{cc}
T_{e e} & T_{e h} \\
T_{h e} & T_{h h}
\end{array}\right)
$$

as well as in site space

$$
\left(\begin{array}{cc}
T_{l l} & T_{l r} \\
T_{r l} & T_{r r}
\end{array}\right)=\left(\begin{array}{cc}
0 & \sigma_{l r} \\
\sigma_{r l} & 0
\end{array}\right)+\left(\begin{array}{cc}
0 & \sigma_{l r} \\
\sigma_{r l} & 0
\end{array}\right)\left(\begin{array}{cc}
g_{l l} & 0 \\
0 & g_{r r}
\end{array}\right)\left(\begin{array}{cc}
T_{l l} & T_{l r} \\
T_{r l} & T_{r r}
\end{array}\right) .
$$

$g$ stays at one site, $\sigma$ hops to the other. Electron-hole conversion for Andreev reflection (AR) is contained in $g . \sigma$ in Eq. (2.4) has no off-diagonal components. In fact, one uses Eq. (2.2) or (2.3) once inserted into itself in the form

$$
T=\sigma+\sigma g \sigma+\sigma g \sigma g T
$$

For our system the transfer function $T$ is produced for each junction separately, so we write $L$ and $I$ or $R$ and $I$ instead of $l$ and $r$. But as the island charge changes with transfers, we denote it at times $\tau$ and $\tau^{\prime}$ as two new indices on $T$ even for times when the particle is in a lead, because any possible following or earlier transfers depend on that (Fig. 3). $T$ will in principle be expanded into a full matrix from any island charge state $n_{1}$ to any other $n_{2}$. However, estimating which excess charges cannot possibly be reached any more with the applied voltage, the matrix is truncated to a finite range, of course. Single hoppings $\sigma$ only mediate between neighboring island charge states. The essence of our Ansatz is the way the $\sigma$ depend on the island charge. Their 
(a)

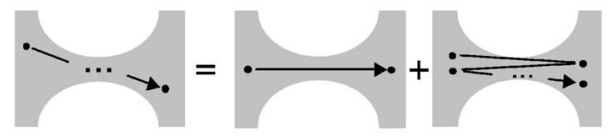

(b)

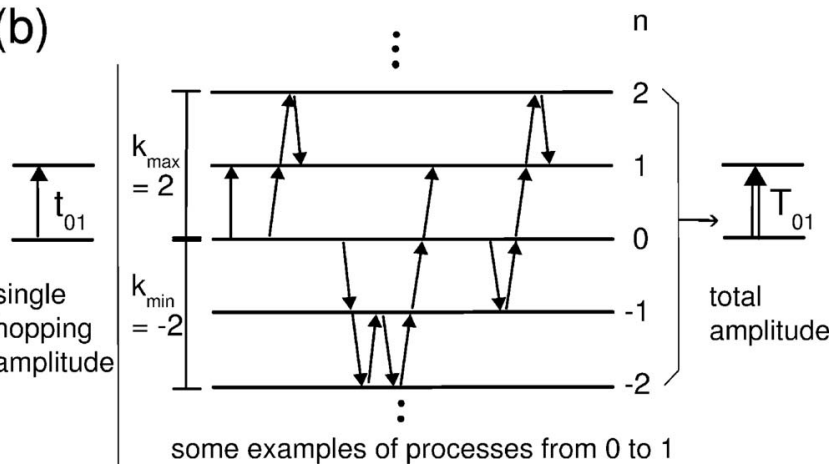

FIG. 3. (a) Illustration of the Dyson equation for the transfer function: Any transfer through a channel is either direct or one back-reflection followed by any transfer ending on the other side. (b) Single hopping with amplitude $t$ from island state 0 to 1 and multiple (Andreev) reflection processes viewed as successive changes of the island charge. All such processes are included in the renormalized transfer amplitude $T$.

phases are determined by the time evolution of the order parameters at the sites. The island with $n$ charges is at potential $\Phi=A-n B$, thus $\Psi_{I}^{n}$ is proportional to $e^{i \tau(-A+n B) / \hbar}, \tau$ denoting the time. The left lead is held at ground potential whence $\Psi_{L}^{n} \sim e^{0}$. Merging an electron (or hole) from the island into the state in the left lead therefore requires one to put the phase of $\left\langle\Psi_{L}^{n}, \Psi_{I}^{n+1}\right\rangle$ into the projection, that is the hoppings (see Appendix A for more detailed explanations):

$$
\begin{gathered}
\sigma_{L I, e e}^{n n+1}=t e^{i \tau[-A+(n+1) B] / \hbar}, \\
\sigma_{I L, h h}^{n n+1}=-t e^{i \tau[-A+n B] / \hbar}, \\
\sigma_{L I, h h}^{n n-1}=-t e^{i \tau[A-(n-1) B] / \hbar}, \\
\sigma_{I L, e e}^{n n-1}=t e^{i \tau[A-n B] / \hbar} .
\end{gathered}
$$

(For $R$ instead of $L$, replace $A$ by $C$. The appropriate value of $t$ has to be used for the respective channel.) The equivalent of Eq. (2.2) for one lead-to-island junction in our case is fully written out and explained in Appendix A. Carrying out the matrix multiplications, the equations separate into two subsets, those containing $T_{L I}$ and $T_{I I}$ as well as those with $T_{I L}$ and $T_{L L}$, respectively. Eliminating $T_{I I}$ from the first set we get

$$
T_{L I}=\sigma_{L I}+\sigma_{L I} g_{I I} \sigma_{I L} g_{L L} T_{L I}
$$

an equation for $T_{L I}$ alone. $T_{L I}^{n_{1} n_{2}}$ will be directly linked to $T_{L I}^{n_{1}-2 n_{2}}$ and $T_{L I}^{n_{1}+2 n_{2}}$. To change from time to frequency (or energy) space we use the Fourier transform (a factor $2 \pi$ is suppressed in the notation)

$$
g\left(\tau-\tau^{\prime}\right)=\int d \omega g(\omega) e^{-i \omega\left(\tau-\tau^{\prime}\right)}
$$

and for $T_{L I}^{n_{1}} n_{2}$ in analogy to Eq. (23) from Ref. 13 we propose the representation (with integers $k$ and $m$ )

$$
\begin{aligned}
& T_{L I}^{n_{1} n_{2}}\left(\tau, \tau^{\prime}\right) \\
& \quad=\sum_{k} \sum_{m} \int d \omega e^{-i \omega \tau} e^{-i k A \tau / \hbar} e^{-i m B \tau / \hbar} e^{i \omega \tau^{\prime}} T_{L I, k m}^{n_{1} n_{2}}(\omega) .
\end{aligned}
$$

The $\sigma$ in Eq. (2.7) show that a multiple (Andreev) reflection across the left junction will change the phase of the order parameter by multiples of $A \tau / \hbar$ and $B \tau / \hbar$. The Fourier transform of $T$ as a function of two time arguments therefore only contains one continuous frequency argument $\omega$, and additionally two integer indices $k$ and $m$. We here determine $T\left(\tau, \tau^{\prime}\right)$ through the functions $T_{k m}(\omega)$. The only important aspect, however, is that the form chosen leaves enough degrees of freedom to make $T\left(\tau, \tau^{\prime}\right)$ satisfy Eq. (2.8). The fraction $A$ of the external voltage dropped over the left junction and the voltage equivalent $B$ of the charging energy will in general be incommensurate. It is helpful to start rather generally with the Ansatz (2.10) and introduce variables $k$ and $m$, although they turn out not to be independent. $k=n_{2}-n_{1}$ and it can be proven that $m=-k n_{1}-k^{2} / 2-1 / 2$, which is an integer, because $k$ in $T_{L I}^{n_{1} n_{1}+k}$ is necessarily odd. In the following, $T$ means $T_{L I}(\omega)$ and $n_{1}$ and $k$ are sufficient as indices. A recursion relation can be established analogously to the singlejunction case

$$
T_{k}^{n_{1}}=\delta_{k, 1} s_{1}+\delta_{k,-1} s_{-1}+\epsilon_{k}^{n_{1}} T_{k}^{n_{1}}+V_{+, k}^{n_{1}} T_{k+2}^{n_{1}-2}+V_{-, k}^{n_{1}} T_{k-2}^{n_{1}+2} .
$$

The only extension to Ref. 13 here consists in solving it for all $n_{1}$ together, because Eq. (2.11) interconnects them in steps of $\Delta n_{1}=2 . s_{1}$ and $s_{-1}$ are the seed to iteratively get $T$ of all $k . s_{1}$ and $s_{-1}$ are just given by single hoppings from Eq. (2.7) and $\epsilon, V_{+}$, and $V_{-}$are products of $g$ functions. The matrices are written out in Appendix A as well as the procedure to solve Eq. (2.11). As the $n$ range, the $k$ range has to be truncated to a finite one from $k_{\min }$ to $k_{\max }$. This does not mean restricting processes to a finite order, but cutting those that reach out by more than $k_{\min }$ or $k_{\max }$ from the initial island charge [see Fig. 3(b), where we chose $\left|k_{\min }\right|=2$ and $\left|k_{\max }\right|=2$ very low just for the simplicity of the drawing].

Before being able to calculate the current, we evaluate the rates by which processes through all channels from both junctions alter the island charge and solve for the probabilities $P_{n}$ to encounter the island with excess charge $n$. The transfer rate through the left junction is given by the trace elements in $e h$ space $^{13}$

$$
\left(\sigma_{L I} G_{I L}^{+-}+\sigma_{I L} G_{L I}^{+-}\right)_{e e} \quad \text { and } \quad\left(\sigma_{L I} G_{I L}^{+-}+\sigma_{I L} G_{L I}^{+-}\right)_{h h}
$$


$G^{+-}$is the Keldysh Green's function built from the advanced and retarded functions by

$$
G^{+-}=\left(\mathbf{1}+G^{r} \sigma\right) g^{+-}\left(\mathbf{1}+\sigma G^{a}\right)
$$

with $g^{+-}=2 i \operatorname{Im} g^{r}$. $G^{r / a}$ is linked to $T^{r / a}$ by $\sigma G^{r / a}=T^{r / a} g^{r / a}$ or $G^{r / a} \sigma=g^{r / a} T^{r / a}$. Equation (2.12) stems from rewriting the current density in terms of the Hamiltonian. Island charge indices on a coupling $\sigma$ can only differ by one and $G^{+-}$, the corresponding matrix element, must connect the initial and final states of that $\sigma$. Terms contained in Eq. (2.12) are therefore naturally recognized as increasing the island charge by one and thus read

$$
\sigma_{L I}^{n+1 n} G_{I L,+-}^{n+1}+\sigma_{I L}^{n+1 n} G_{L I,+-}^{n n+1}
$$

or decreasing it by one (replace $n+1$ by $n-1$ ). We note that only rate terms changing the island charge by one exist in the form (2.14) and have to be taken into account. MARs are included. A MAR contributes to all rates, each changing the island charge by one, between its initial and final island charges. This will be explained further when rewriting the rates in terms of the $T$ and when reanalyzing them in Sec. IV.

In order not to break the coherence of multiple (Andreev) reflection processes the rate to increase the island charge from $n$ to $n+1$ has to be counterbalanced by the one to decrease it from $n+1$ to $n$ through the same channel before entering it into the rate matrix. So, instead of Eq. (2.14) and the analoguous term with $n+1$ replaced by $n-1$ we only have rate terms of the form

$$
\sigma_{L I}^{n+1 n} G_{I L,+-}^{n n+1}+\sigma_{I L}^{n+1 n} G_{L I,+-}^{n n+1}-\sigma_{L I}^{n n+1} G_{I L,+-}^{n+1 n}-\sigma_{I L}^{n n+1} G_{L I,+-}^{n+1 n} .
$$

As illustrated in Fig. 4, Eq. (2.15) is understood as the rate $R_{n}^{n+1}$ from $n$ to $n+1$ if it is positive and as the (positive) rate $R_{n+1}^{n}$ from $n+1$ to $n$ if it is negative. The idea behind using such net rates without weighting the positive and negative terms in Eq. (2.15) by the probabilities $P_{n}$ and $P_{n+1}$, respectively, is the following: In case that the $n \rightarrow n+1$ contribution from Eq. (2.14) exceeds the $n+1 \rightarrow n$ contribution, it means that a certain percentage of all charges passing

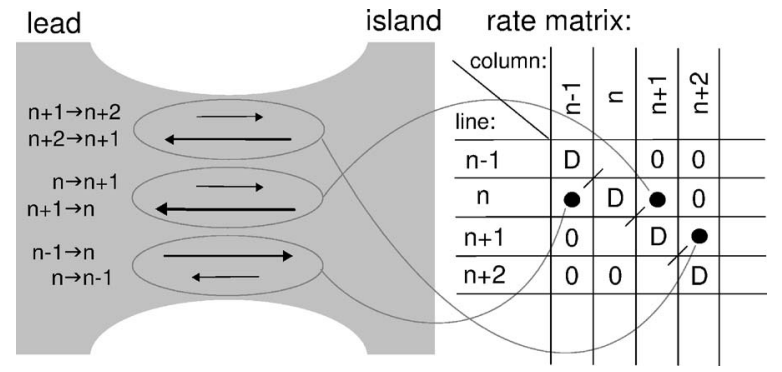

FIG. 4. Composing net rates through a channel between neighboring island charge states and places where to enter them in the rate matrix. Here, for example, the net rate between $n+1$ and $n+2$ is from $n+2$ to $n+1$ and goes on the upper side diagonal, same as the net rate between $n$ and $n+1$. Between $n-1$ and $n$, the net flow is from $n-1$ to $n$ and goes on the lower side diagonal. Only the three middle diagonals are nonzero. The diagonal entries $D$ are determined after all other entries have been filled in. Other channels from the same junction as the one drawn or from the other junction may fill the fields still empty here or add to the ones with as well. However, a channel can only contribute to one entry of each pair marked with $/$.

through the regarded channel in an $n \rightarrow n+1$ step is immediately back-reflected as an $n+1 \rightarrow n$ step without letting the island actually come to the $n+1$ state. An analogous argument holds for the $n+1 \rightarrow n$ part outweighting the $n \rightarrow n+1$ part. In fact, our construction of the rate matrix allows a single step out of a MAR that changes the island charge between $n$ and $n+1$ to contribute to real current flow, with the other steps of the MAR only happening virtually. This conserves coherence and interference between our basis states. From a mathematical point of view, taking expression (2.15) ensures that entries of the rate matrix are real. In contrast to Eq. (6) from Ref. 13, with the plus sign and the island charge indices on $\sigma$ and $G$, Eq. (2.14) is not real. However, it can be shown that Eq. (2.15) is composed of pairs of complex conjugate terms and equal to twice the real part of the first two products. Using $T(\omega)$ made before, Appendix B shows that Eq. (2.15) can be calculated as

$$
\begin{aligned}
2 \operatorname{Re} \operatorname{Tr}\left(\sigma_{L I}^{n+1 n} G_{I L,+-}^{n n+1}+\sigma_{I L}^{n+1 n} G_{L I,+-}^{n n+1}\right)= & 2 \operatorname{Re}\left\{\int d \omega \sum_{k}\left[g_{I I}^{r}(\omega) T_{I L, k}^{n-k, r}(\omega) g_{L L}^{+-}(\omega+k A+m B) T_{L I, k}^{n-k n, a}(\omega)\right]_{h h}\right. \\
& +\int d \omega \sum_{k}\left[T_{L I, k}^{n+1 n+k+1, r}(\omega) g_{I I}^{+-}(\omega) T_{I L, k}^{n+k+1 n+1, a}(\omega) g_{L L}^{a}(\omega+k A+m B)\right]_{h h} \\
& +\int d \omega \sum_{k}\left[T_{I L, k}^{n+1 n+1-k, r}(\omega) g_{L L}^{+-}(\omega+k A+m B) T_{L I, k}^{n+1-k n+1, a}(\omega) g_{I I}^{a}(\omega)\right]_{e e} \\
& \left.+\int d \omega \sum_{k}\left[g_{L L}^{r}(\omega+k A+m B) T_{L I, k}^{n+k, r}(\omega) g_{I I}^{+-}(\omega) T_{I L, k}^{n+k n, a}(\omega)\right]_{e e}\right\} .
\end{aligned}
$$


Both charge indices on $T$ are written out for clarity; $m$ is determined by the first one and $k$ in each case. Only $T_{L I}$ must be calculated by recursion from Eq. (2.11); $T_{I L}$ is then easily inferred as given in Appendix B. For the rate between $n$ and $n+1$ in Eq. (2.16) the outer indices are $n$ or $n+1$; however, the sums are over all $k$, inner indices can thus be any charge number. Here is thus seen the way interference between charge states enters the formalism. The $\omega$ integration must be done numerically.

The rates for all channels from both junctions are added ${ }^{23}$ on the upper and lower side diagonal of the rate matrix (Fig. 4). In a single junction the contributions of individual channels are independent and are not mixed by MARs. ${ }^{12}$ In our case the mutual influence between different channels is treated classically through the changing island charge and potential. The presence of the second junction constitutes additional channels, in the same way as more channels in one contact would do. The diagonal elements in our rate matrix are given by the negative sum of the two elements above and below in the same column. The stationary state requirement for the probabilities $P_{n}$ of the island charge states, which can be gathered into the vector $\vec{P}$, reads $d \vec{P} / d t=0$ or

$$
\begin{gathered}
\left(\begin{array}{cccc}
\cdots & & & \\
& -R_{n-1}^{n-2}-R_{n-1}^{n} & R_{n}^{n-1} & 0 \\
R_{n-1}^{n} & -R_{n}^{n-1}-R_{n}^{n+1} & R_{n+1}^{n} & \\
0 & R_{n}^{n+1} & -R_{n+1}^{n}-R_{n+1}^{n+2} & \\
& \\
& \times\left(\begin{array}{c}
\ldots \\
P_{n-1} \\
P_{n} \\
P_{n+1} \\
\ldots
\end{array}\right)
\end{array}\right)=\left(\begin{array}{c}
\ldots \\
0 \\
0 \\
0 \\
\ldots
\end{array}\right) .
\end{gathered}
$$

Of course, for the numerical evaluation, the dimensions of the rate matrix and the $\vec{P}$ vector are cut to a finite range from $n_{\min }$ to $n_{\max } \cdot R_{n}^{n-1} P_{n}$ is the flow going out from state $n$ to $n-1, R_{n}^{n+1} P_{n}$ the one going to $n+1$. Thus, these terms are entered on the respective upper and lower side diagonals for they increase $P_{n-1}$ and $P_{n+1}$, and with negative sign on the main diagonal because they diminish $P_{n}$. In each line, the $n$ th, for example, the outgoing flow $\left(-R_{n}^{n-1}-R_{n}^{n+1}\right) P_{n}$ from state $n$ has to be compensated by the incoming flow $R_{n-1}^{n} P_{n-1}+R_{n+1}^{n} P_{n+1}$ to state $n$ from neighboring states. The linear system (2.17) is easily solved for the $P_{n}$, imposing a common scaling factor by $\Sigma_{n} P_{n}=1$. From Eq. (2.17) it can also be seen that for a current flow, at least for one $n$ there has to be a rate contribution in the $n \rightarrow n+1$ direction from some channel and a contribution in the $n+1 \rightarrow n$ direction from another. Otherwise all but one of the $P_{n}$ will be zero and the island gets blocked in a particular state. For a net dc current the two mentioned channels have to belong to different junctions, of course. As in the stationary state charge must not accumulate on the island, the dc current is the same through both junctions. It can be calculated for any of the two junctions (the right $R \leftrightarrow I$, for example). Here, in contrast

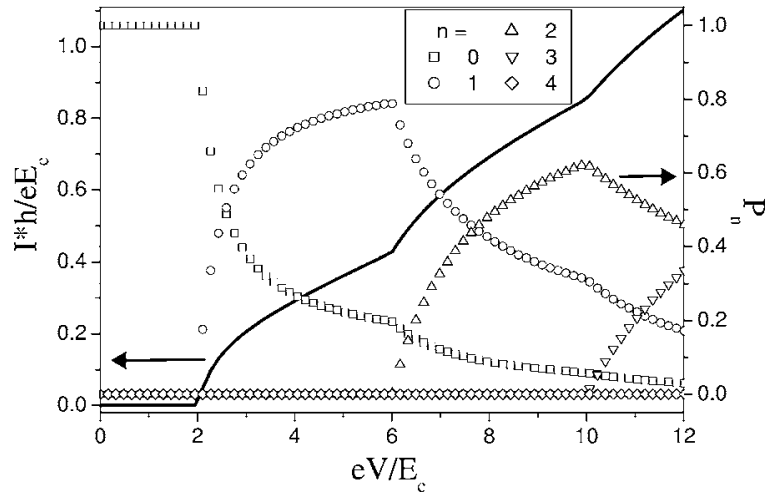

FIG. 5. Current $I$ and island charge state probabilities $P_{n}$ as a function of voltage for $\Delta=0$ (normal state), $C_{1}=C_{2}, U=0$, one channel per junction, left $\theta_{1}=0.48$, right $\theta_{2}=0.06$.

to the rate matrix, only the rates for one junction are used and they are taken with signs according to the direction of the current flow

$$
I=e \sum_{n}\left(R_{n R \leftrightarrow I}^{n+1}-R_{n R \leftrightarrow I}^{n-1}\right) P_{n}
$$

( $e$ is the elementary charge unit and the $R$ are in $s^{-1}$ ).

\section{RESULTS AND DISCUSSION}

We start by evaluating our model for some well-studied situations. In the limit $\Delta=0$ we recover the normal state behavior. The current-voltage $(I-V)$ curve is exactly the same as described by orthodox theory, a fact that can serve as a consistency test. For vanishing gate voltage and equal junction capacitances the current sets in after the Coulomb blockade regime at $e V=2 E_{c}$ and increases with discontinuous derivative at $e V=(4 n+2) E_{c}$ (Fig. 5). At these voltages the next higher island charge state can be reached which is also seen in the $P_{n}$. For unequal capacitances the thresholds will depend on the ratio $C_{1}: C_{2}$. In a plot of $I$ versus both $V$ and $U$ the edges would form Coulomb diamonds. For only slightly different transmissions of the two junctions the island also goes into states with negative $n$ during charge transport.

In order to compare our algorithm with results for a single junction in the superconducting state [Fig. 6(a)] we choose high junction capacitances such that the island charging energy $E_{c}$ is much less than the gap $\Delta$ and not the dominant parameter. For one junction with one perfectly open channel $\left(\theta_{1}=1\right)$ and the other with one channel of medium transmission $\left(\theta_{2}=0.48\right)$, there is a steplike structure in the current, resembling the single-junction characteristic. Due to the fact that, in contrast to a lead, a junction with $\theta=1$ means a series resistance of $13 k \Omega$, the single-junction characteristic is not exactly reproduced, however. The steps are shallower and the ohmic behavior is only observed well above $2 \Delta$. The step positions are not necessarily simply fractions of $4 \Delta$ (see below). For the combination of a channel with perfect transmission $\left(\theta_{1}=1\right)$ in one junction and a channel of small transmission $\left(\theta_{2}=0.06\right)$ in the other junction, the result is essentially a product of the throughputs of the two junctions 

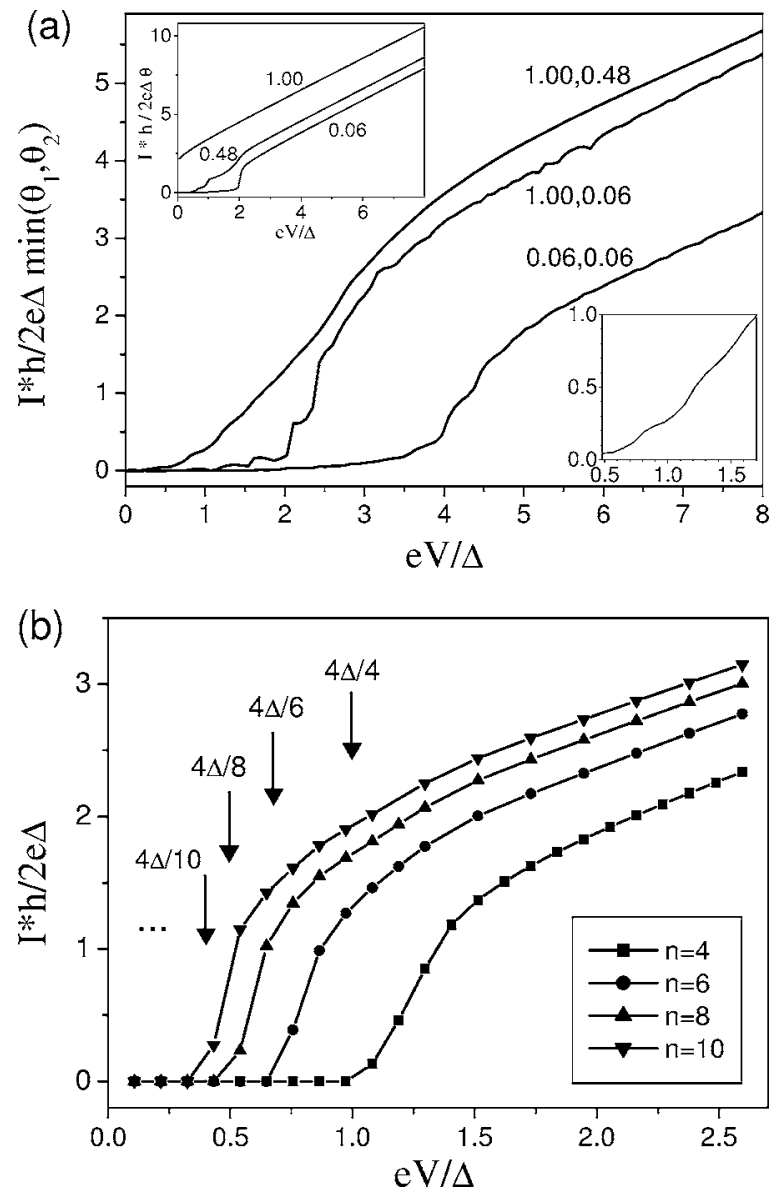

FIG. 6. (a) $I-V$ for $E_{c} / \Delta=0.1, C_{1}=C_{2}, U=0$, different $\theta_{1}$ and $\theta_{2}$ [note that division by $\min \left(\theta_{1}, \theta_{2}\right)$ to get comparable $I$ values is not a full normalization for the double junction]. Lower inset: Magnification of the low-voltage regime for $\theta_{1}=1.00$ and $\theta_{2}=0.48$. Upper inset: $I-V$ of a single junction for three values of $\theta$ (Ref. 13). (b) Test $I-V$ curves for $U=0, \theta_{1}=\theta_{2}=1$, and $E_{c}=0$ with increasing the considered range of island charge states $\left[n_{\min }, n_{\max }\right]=[-n, n], k_{\max }$ $=n$ [symbols are drawn because of the coarser point density and larger scale than in (a) here].

with main current onset at about $e V=2 \Delta$, as expected. This curve appears noisier than the one above it, due to the division of $I$ by $\min \left(\theta_{1}, \theta_{2}\right)$ for plotting. In the case of two junctions with low transmission $\left(\theta_{1}=\theta_{2}=0.06\right)$ a voltage of $e V$ $=4 \Delta$ is required to get non-negligible current, that is a potential difference of $2 \Delta$ per junction. The current rise is shallower, though, than the steep onset for a single junction or a double tunnel junction, ${ }^{20,24}$ in agreement with preliminary experimental results ${ }^{17}$ on a setup replacing at least one contact by a few-channel break junction. The influence of the charging energy $E_{c}$ is still present in Fig. 6(a) with $E_{c} / \Delta$ $=0.1$. Further reducing this ratio would require us to enlarge the number of included island charge states accordingly and thus by much increase the calculation time on a PC. In Fig. $6(\mathrm{~b})$ with $\theta_{1}=\theta_{2}=1$ the charging energy (parameter $B$ ) has been artificially set to zero. As we are thus deprived of any means to reduce the amplitude to charge the island to ever higher $n$ with MARs of increasing order, a correct result can only be obtained in the limit $\left[n_{\min }, n_{\max }\right] \rightarrow[-\infty, \infty]$. With
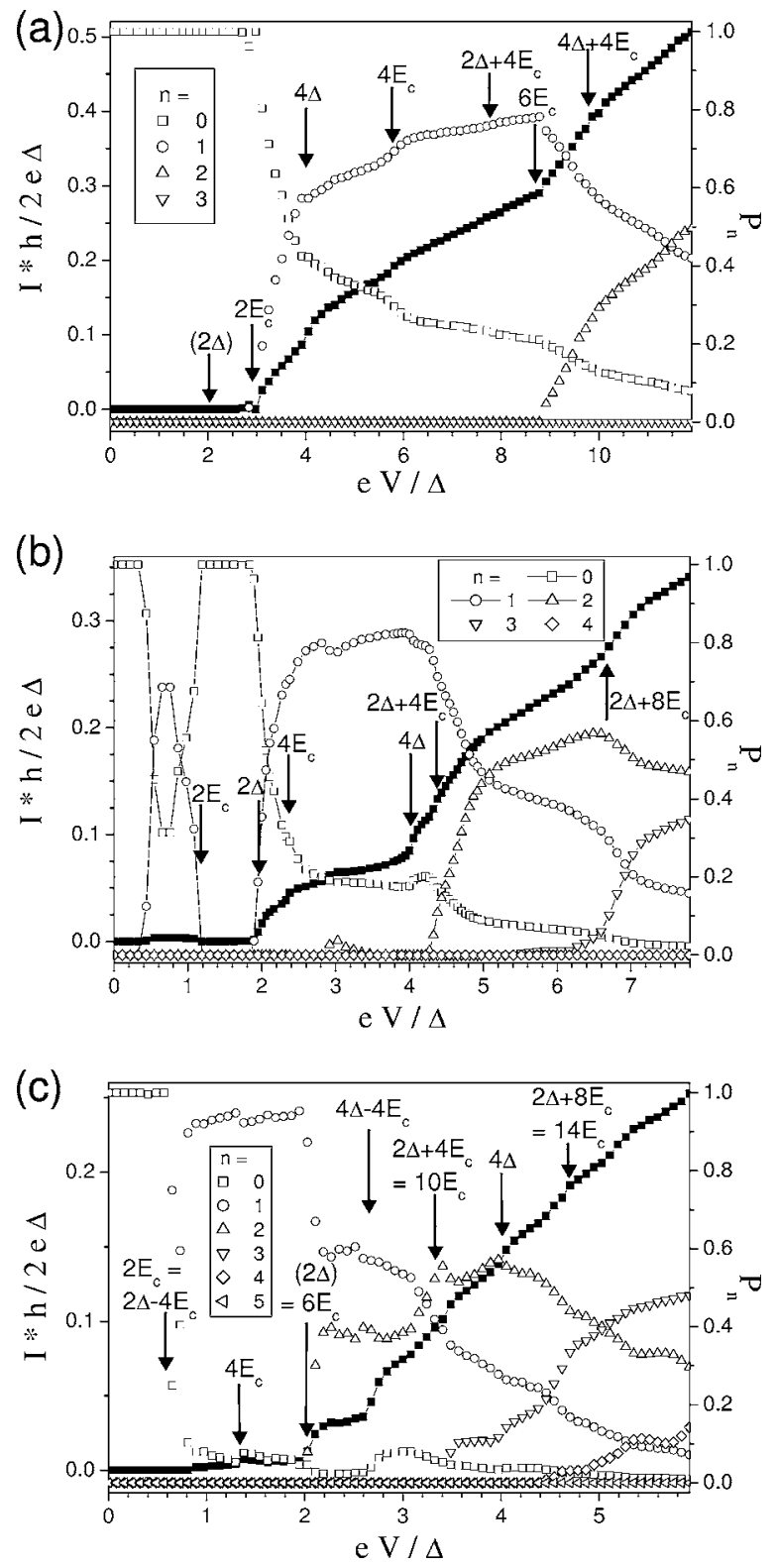

FIG. 7. $I-V$ and corresponding $P_{n}$ for $U=0, \theta_{1}=0.48$ and $\theta_{2}$ $=0.06, C_{1}=C_{2}$ and (a) $E_{c} / \Delta=1.44$, (b) $E_{c} / \Delta=0.58$, (c) $E_{c} / \Delta$ $=0.34$.

$E_{c}=0$ and $\theta_{1}=\theta_{2}=1$ there is one steep current onset without further steps in the curve. In the single-junction calculations in Ref. 13 for all $\theta<1, I=0$ at $V=0$, while $I / 2 \Delta=2 e / h$ at $V=0$ for $\theta=1$. Our model in contrast produces onsets approaching $V=0$ with enlarging the $n$ range. The limited $n$ range will always prevent our double-junction algorithm from giving a finite $I$ arbitrarily near $V=0$. The qualitatively correct behavior for high transmissions is nevertheless demonstrated by the curves from Fig. 6 .

In Fig. 7 we show $I-V$ curves together with island charge state probabilities for one junction with a channel of medium transmission $\left(\theta_{1}=0.48\right)$ and the other with a channel of low transmission $\left(\theta_{2}=0.06\right)$, which is, however, meant to be an arbitrary example. We always choose zero gate voltage and equal junction capacitances, but different ratios of $E_{c}$ and $\Delta$. 

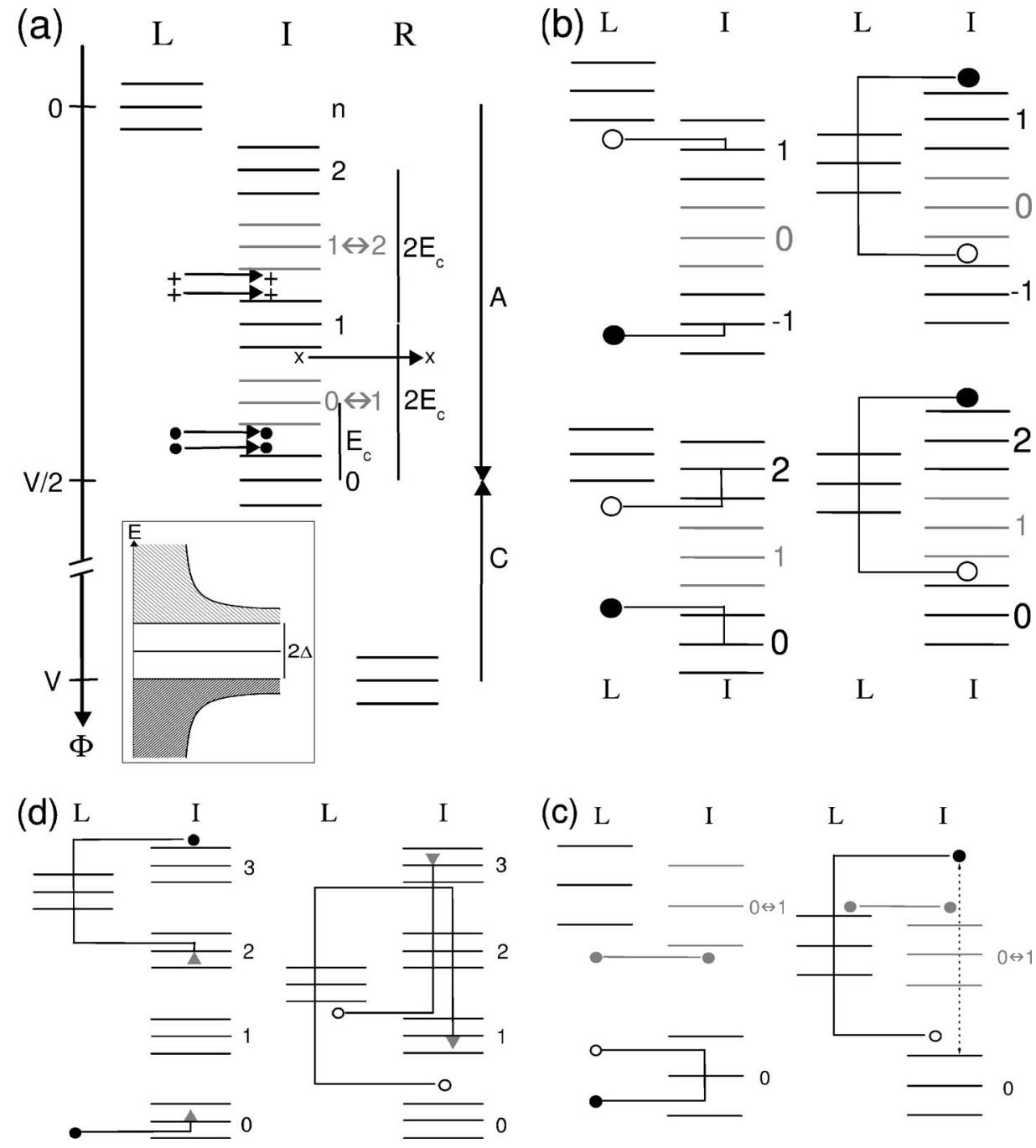

FIG. 8. (a) Potential levels of left $(L)$ and right $(R)$ leads and island $(I)$ with different excess charges. Every line triplet marks a density of states as in the inset with a $2 \Delta$-wide gap, occupied/empty electron/hole states below and vice versa above. The gaps of different island charge states may be overlapping or separated (as drawn) depending on the ratio $E_{c} / \Delta$. The 0 state of the island is at $V / 2$ in case that $C_{1}$ $=C_{2}$ and $U=0$, or else its distance from the potential of the left lead in the direction of the arrow is given by $A$ and its distance from the right-lead Fermi level by $C$ (see text). Also drawn are "charge-changing" states (gray) of the island midway between states $n$ and $n+1$. Horizontal arrows mark the conditions for charging the island from 0 to $1(\mathbf{)})$ and from 1 to $2(+)$ from the left as well as decharging it from 1 to 0 by releasing one electron to the right $(\times)$, all by single electron transfers. (b) A first-order AR in the left junction including charging the island from 0 to 1 at some stage can be an electron from the left encountering the island in state -1 , being Andreev reflected there and thus leaving it in state 1 when returning to the left as a hole, an electron from the left coming onto the island in state 0 and leaving it as a hole in state 2 , a hole from the island in state -1 going to the left and after AR there coming back as an electron to final state 1 , or a hole leaving the island from state 0 and being back as an electron in final state 2. (c) Shortcut versions to derive conditions from the energy level diagram for the four processes from (b) to give a net contribution to charge the island from 0 to 1. (d) Two examples of second-order AR processes across the left junction. The full processes would go from $n=0$ to $n=3$. For the shortcut version to derive the lowest threshold for all second-order AR processes together to contribute to an island charging rate from 0 to 1 , see the inset of Fig. 13; this has to be complemented by the same charging condition (gray dot and arrow) as in the left diagram from (c).

The observed current onsets in the following will be correlated to particular transport processes. In all three cases, but most pronounced in Fig. 7(b), there is a current increase at $V / 2=2 \Delta / e$ or $e V=4 \Delta$. This is when an electron from the left lead finds an empty state at its energy level on the island, initially with zero excess charge, and can therefore hop over [see Fig. 8(a)] and shift the island to charge one. However, this possibility is coupled to the condition that the voltage also exceeds $2 E_{c}$ or $V / 2>E_{c} / e$, which it does in our ex- amples because $4 \Delta>2 E_{c}$ for all of them. The charging energy requirement corresponds to checking that the upper rimp of the occupied electron reservoir of the island at potential midway between charge zero and one, levels with occupied electron states in the left lead. The electron relaxed into the electron sea below the gap may have come from the left. The meaning of the midway potential can be understood by considering that the charging energy $e^{2} / 2 C$ equals only half the voltage difference between successive charge states 
$\delta \Phi=e / C$ times $e$. It will be shown in the next section how the dual threshold, $4 \Delta$ and $2 E_{c}$ here, emerges from the rates derived in Sec. II. The particle paths in the energy-level picture in Fig. 8 are an interpretation, not premises of our model. For comparison, remark that the orthodox theory model yields $4 \Delta+2 E_{c}$ as the threshold for single-charge hopping (at $U=0$ ), which is indeed verified in experiments on double tunnel junctions; the onset can be brought down to $4 \Delta$ by a gate voltage. ${ }^{20,24}$ In our calculations, for the case that the onset is at $4 \Delta$ at $U=0$, it comes down to $4 \Delta-2 E_{c}$ with the appropriate gate voltage, and down to zero if the onset is at $2 E_{c}$ for $U=0$. Our model predicts thresholds which do not coincide with those obtained from OT by classical energy balance arguments alone. It thus suggests that the distinction between tunnel junctions and quantum-point connections may not merely consist in the latter having higher transmission, but in a qualitatively different interaction linking both sides of the contact. Transport characteristics of a single junction without the interplay of the superconductor gap and the charging energy of an island, could, however, not reveal such a property.

We now go on with the discussion of the results from our model. All essential features can already be seen for $C_{1}$ $=C_{2}$ and $U=0$ whence we mostly restrict ourselves to that special case here. Analogously to the conditions for charging the island from zero to one, for charging it from one to two by single-charge transfer requires $\mathrm{eV}>4 \Delta+4 E_{c}$ and $e V$ $>6 E_{c}$. Electrons from below the gap in the left lead have to find empty states above the gap in the island's $n=1$ state and the lower gap edge of the midway state between one and two has to be below the lower gap edge left [see Fig. 8(a)]. Again, $4 \Delta+4 E_{c}>6 E_{c}$ is valid for all examples in Fig. 7. (In writing down voltage conditions we will skip the obvious factor $e$ from now on.) Evidently, as seen from the current and the $P_{n}$, the island is charged to one or even higher states well below $4 \Delta$ in all cases in Fig. 7, which, of course, is due to Andreev reflection (AR). For lowest-order AR, there are four processes depicted in Fig. 8(b), that-when decomposed into two charging steps-contain a step from $n=0$ to $n=1$. For the two processes beginning with sending an electron out from an occupied state on the left, it does not matter whether this electron comes onto the island in its initial state above or below the Fermi level or inside or outside the gap. The reflected hole energetically lies at the potential mirrored at the Fermi level, the mirrored half of the process, however, drawn at the final island state with $n$ increased by two. The AR only occurs if this mirrored level corresponds to filled electron or empty hole states in the left lead, that is lies below the gap on the left. This requirement leads to the condition $V>2 \Delta$ for the AR from -1 to 1 and to $V>2 \Delta+4 E_{c}$ for the AR from 0 to 2 , respectively. As the left lead is held at fixed potential, for the two processes starting with a hole on the island and the AR happening in the lead, no shift of the mirrored half is necessary. The reflected electron, however, has to find an empty level in the final island state with $n$ increased by two compared to the initial state. Again, the AR from -1 to 1 requires $V>2 \Delta$ and the one from 0 to 2 needs $V>2 \Delta$ $+4 E_{c}$.

Even if the AR between -1 and 1 contributes to charging the island up from 0 to 1 , for the same voltage $V$ there may still be AR in the opposite direction from 2 to 0 including a decharging step from 1 to 0 through the same transport channel. A net island charge increase from 0 to 1 from the two processes from Fig. 8(b) with AR on the island is found only if $V>2 E_{c}$. The proof will be given in the next section. The shortcut version how to derive from the energy-level diagram the lowest threshold for first-order AR to bring the island from $n=0$ to $n=1$ together with the additional charging energy condition $V>2 E_{c}$ is shown in Fig. 8(c). The electron from the left enters the island in the $n=0$ state and is mirrored at the Fermi level there without being shifted to a higher island charge state. Requiring an empty level for the reflected hole on the left necessitates $V>2 \Delta$. As for singlecharge transfers we now additionally demand that the upper rimp of the filled electron levels of the $0 \leftrightarrow 1$ change state lies below the lower gap edge of the left lead, which means that $V>2 E_{c}$. The complementary process with AR in the lead is just drawn for completeness and gives the same thresholds. Require an empty level for the reflected electron also in the island $n=0$ state where the initial hole came from. And here demand that the lower edge of the filled hole states in the left lead is above the gap of the island $0 \leftrightarrow 1$ change state. In conclusion the onset of the discussed first-order AR processes is found at $V=2 \Delta$ or $V=2 E_{c}$, according to which of the two represents the larger threshold. Figure 7(b) corresponds to the first, Fig. 7(a) to the second case. In Fig. 7(c), the step at approximately $2 \Delta$, which happens to be nearly equal to $6 E_{c}$ in this case, is due to charging to the $n=2$ state by higher-order MARs, requiring $4 E_{c}$ plus only a fraction of $\Delta$. The reason why there is (almost) no charging from $n=0$ to $n=1\left(P_{0} \approx 0\right)$ despite $2 E_{c}<2 \Delta$ will be understood in context with the decharging condition (see below). The shortcut process diagrams from Fig. 8(c) can also be interpreted in the peculiar way that in our model it is possible to add a single charge to the island by AR. Another charge may go off to the other lead before the second charge comes from the AR, thus opening a way to get a current contribution from such a partial Andreev reflection. An equivalent description would be that the second transfer of the AR only happens virtually. Even if the transfer charging up the island belongs to an AR, only the charging energy condition for one additional charge has to be fulfilled. That is $V>2 E_{c}$ for, for example, bringing the island from $n=0$ to $n=1$ by the first half of the AR from 0 to 2 . If the second part of this AR actually carries the island from $n=1$ to $n=2$, the charging condition reads $V>6 E_{c}$ just like for the single-charge transfer.

A deeper analysis of the rate contributions from singlecharge transfers such as the above for lowest-order AR on the island reveals that the dual threshold there is also due to the counterbalance of several processes. There are two possibilities to increase the island charge from $n=0$ to $n=1$. An electron from the left lead can go into the final island state $n=1$ for $V>4 \Delta+4 E_{c}$. A hole from the initial island state $n$ $=0$ can already find an empty level in the left lead for $V$ $>4 \Delta$. If $V<4 E_{c}-4 \Delta$, the island in the $n=1$ state can, however, even release an electron to the left lead. A net flow from $n=0$ to $n=1$ is obtained for $V>2 E_{c}$, hence the dual threshold $V>4 \Delta$ and $V>2 E_{c}$, which will also be shown to follow from Eq. (2.16) in the next section.

Figure 8(d) sketches two second-order AR processes from the initial state $n=0$ to final state $n=3$. The shortcut diagram 


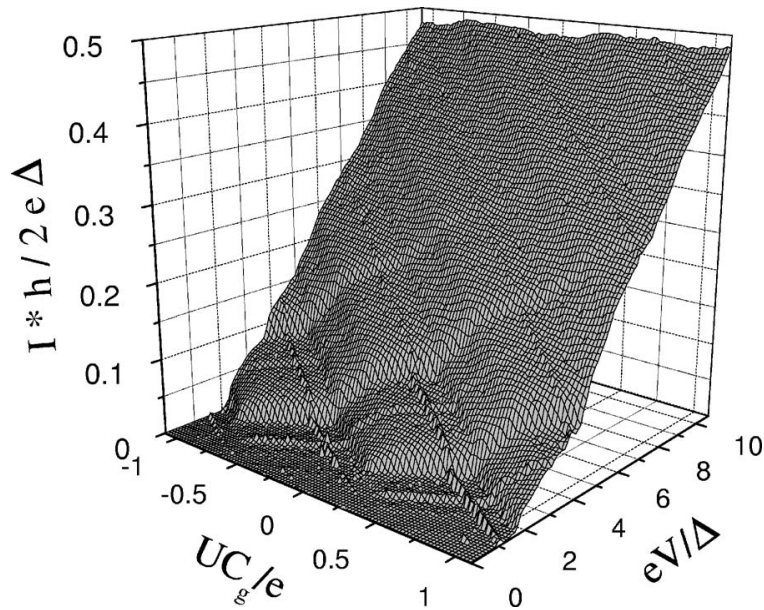

FIG. 9. Current as a function of transport and gate voltage for the same junction transmissions as in Fig. 7, $C_{1}=C_{2}$ and $E_{c} / \Delta$ $=0.7$.

to deduce the threshold from which on all second-order AR containing a $0 \rightarrow 1$ step contribute to the $0 \rightarrow 1$ charging rate is given in the inset of Fig. 13. This diagram serves as an example to explain the geometric derivation of a voltage threshold in detail. The process starts in the electron reservoir of the left lead at some energy level lying an amount $x$ below the gap, that is at potential $\Delta+x$. The particle enters the island and is Andreev reflected at the Fermi level of the 0 state. That brings it to potential $V / 2+(V / 2-\Delta-x)$. It now travels back to the left lead and is again Andreev reflected there, ending up at $-V+\Delta+x$. To find an empty electron state at this energy on the island-still in the 0 state-requires $-V+\Delta+x<V / 2-\Delta$ or, as $x$ may be infinitely small $V$ $>4 \Delta / 3$, that is as expected twice the threshold for secondorder AR from the single junction. In addition to that condition, $0 \rightarrow 1$ charging by second-order $\mathrm{AR}$ at the same time requires $V>2 E_{c}$.

For current flow through the whole system, when increasing the island charge via the left junction, decharging the island via the right junction must be allowed. Changing from $n=1$ to $n=0$ by single-electron transfer means that an electron from the occupied states below the gap on the island in state 1 has to find an empty state above the gap in the right lead, requiring that $V / 2-2 E_{c}+\Delta<V-\Delta$ or $V>4 \Delta-4 E_{c}$ [Fig. 8(a)]. For a junction of low transmission single-charge hopping is the essential transfer process. Higher-order processes are included in our model the same way as for the left junction, but we mostly neglect them for the interpretation of the dominating features in the $I-V$ curves from Fig. 7 because we chose the second junction of low transmission there. In the examples from Figs. 7(a) and 7(b), $4 \Delta-4 E_{c}$ is less than $2 E_{c}$ or $2 \Delta$, respectively, thus in the voltage region following the main onset, current flow takes place by charging the island from 0 to 1 via the left junction by partial AR and decharging it from 1 to 0 via the right junction by singleparticle transfer. For higher voltages the oscillation of the island state shifts to alternating most of the time between state 1 and state 2, and so on. In Fig. 7(c) the first step marking the main onset at $6 E_{c}$ is made by a process involving states 1 and 2. Even if in this example $2 \Delta>2 E_{c}$ and

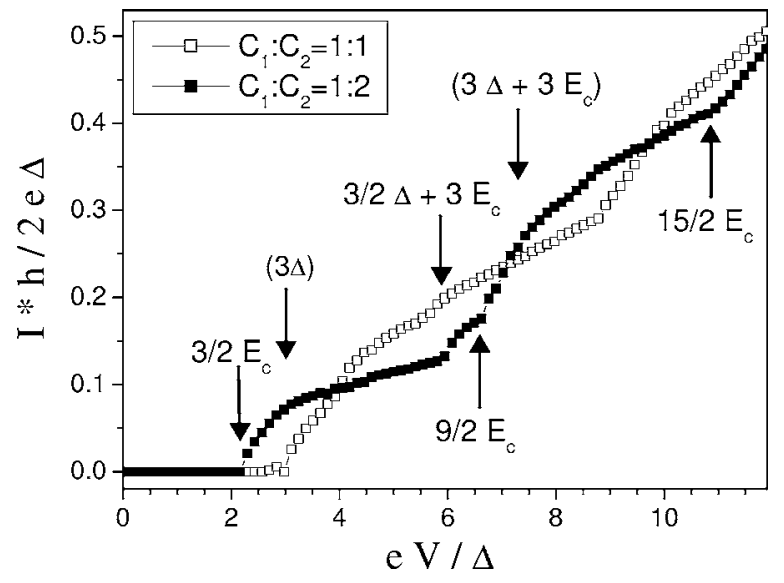

FIG. 10. $I-V$ for the same parameters as in Fig. 7(a), especially same $E_{c}$, but $C_{1}: C_{2}=1: 2$ (full symbols). The curve from Fig. 7(a) is plotted again for comparison (open symbols).

charging from 0 to 1 by partial AR would be possible, this cannot yet lead to a current flow, because the decharging process from 1 to 0 across the right junction is suppressed. However, decharging from 2 to 1 is allowed if $V>4 \Delta-8 E_{c}$, which is below $6 E_{c}$ in the example. Island state 0 comes into play with a pronounced step in the $I-V$ at $4 \Delta-4 E_{c}$, but at slightly further increased voltage higher states are adopted much more frequently because of the low $E_{c}$ in this case.

In Fig. 7(c) there is a small current contribution starting right above $2 E_{c}$ and making the island switch between states 0 and 1 . The origin of this must be a partial higher-order AR requiring a voltage equal to only a fraction of the gap $(4 \Delta / 5$ would fit here) to charge the island up across the left junction. A small rate for decharging across the right junction also by partial (here first-order) AR, allowed above $2 \Delta$ $-4 E_{c}$, is seen in this case. The island almost gets trapped in state 1 , though. The little current bump at low voltages in Fig. 7(b) is of more subtle origin. The island is charged to 1 even below $V=2 E_{c}$. Although only island states 0 and 1 are actually adopted, the charging rate must be due to interference with higher excess charge states. In double tunneljunction experiments so-called Andreev and Josephson quasiparticle cycles also appear as ridges in the CB regime ${ }^{20,24}$ and have been explained by coupling between island charge states. $^{25,26}$ In the next section we shall give an idea how contributions violating the simple charging energy conditions used before may arise in our model and why they are valid.

Figure 9 illustrates that the edges in the $I-V$ characteristic, although not all equidistant as in the normal-conducting case, with varying gate voltage form Coulomb diamonds. The asymmetry in the shape of the steps in the two diagonal directions along the edges is due to the unequal junction transmissions $\theta_{1} \neq \theta_{2}$. In the general case where $C_{1} \neq C_{2}$ and $U \neq 0$, in the conditions involving the left junction, $V / 2$ has to be replaced by $A$ and in those for the right junction there is $-C$ instead of $V / 2$. Figure 10 shows that edges in the $I-V$ curve are no longer found at even multiples of $\Delta$ and/or $E_{c}$ for unequal capacitances. With the simple ratio $C_{1}: C_{2}$ $=1: 2$ that we chose as an example, the values can still be given in fractions of $\Delta$ and $E_{c}$, though. The shift of edge positions with $C_{1}: C_{2}$ is an effect that is already present in the 


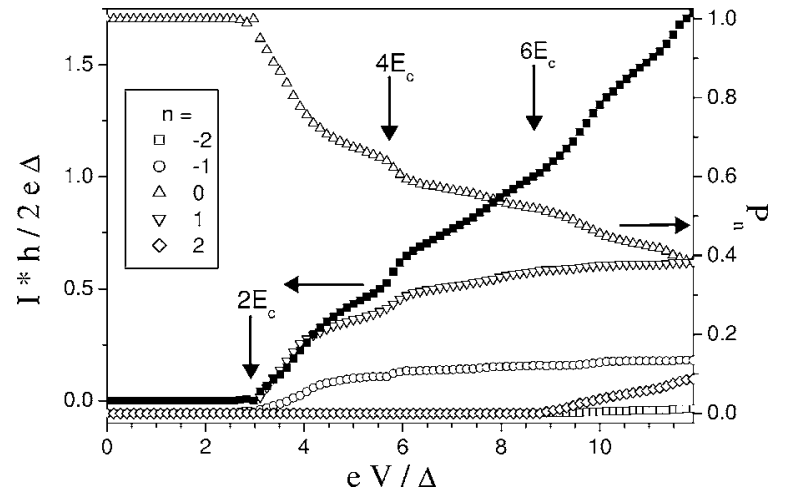

FIG. 11. $I-V$ and $P_{n}$ for the same parameters as in Fig. 7(a), except that here $\theta_{2}=0.3$.

normal state and OT, of course. The example in Fig. 11 demonstrates that the island passes through states with negative as well as positive excess charge if the transmissions of the two junctions are of comparable size. In this situation also the counterparts of the transport processes discussed above with the roles of the junctions as well as electrons and holes interchanged contribute to the transport. The distinct current rise at $4 E_{c}$, also present in Fig. 7, can be assigned to the AR process between $n=-1$ and $n=1$ with the AR taking place in the lead [see Fig. 8(b) and next section]. The contribution of this process is not necessarily zero for lower voltages, however, a diagram analogous to Fig. 15 would reveal a singularity matching at $V=4 E_{c}$. In Fig. 12 we show an example with two channels in the left junction, and for comparison the $I-V$ 's with only one channel present. As charge transports through the two channels have to share the throughput of the one channel in the right junction, the current is less than the sum of both single-channel setups. This obvious phenomenon is also found in OT models.

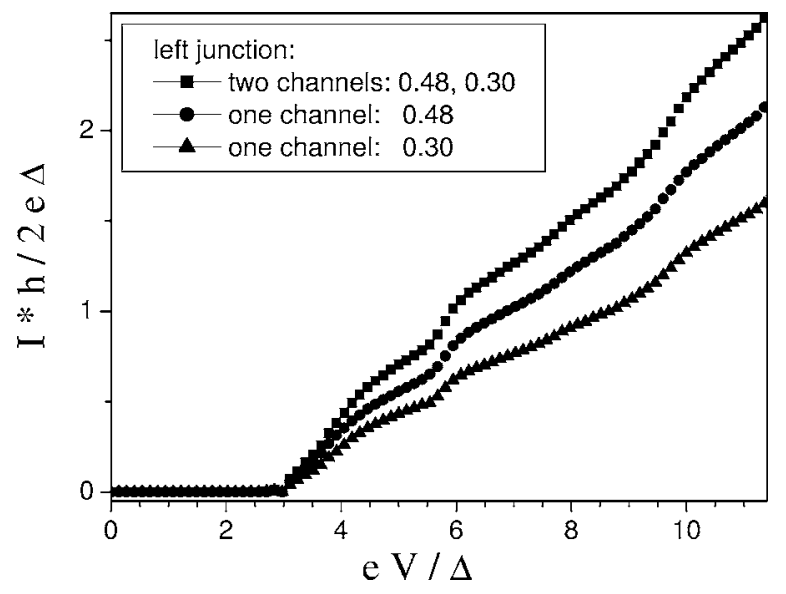

FIG. 12. $I-V$ for $U=0, C_{1}=C_{2}, E_{c} / \Delta=1.44$, one channel with $\theta_{2}=0.48$ in the right junction and three different configurations of $\theta_{1}=0.3, \theta_{1}=0.48$ and two channels with $\theta_{11}=0.48$ and $\theta_{12}=0.3$ in the left junction.

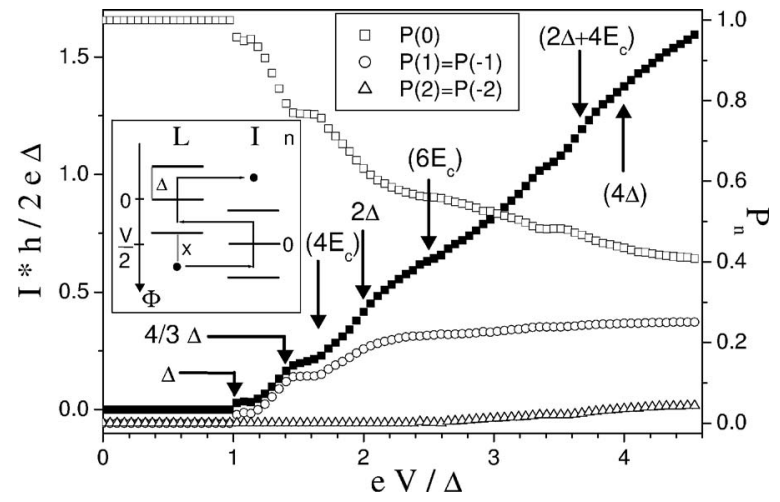

FIG. 13. $I-V$ for $U=0, C_{1}=C_{2}, E_{c} / \Delta=0.42$, one channel per junction with $\theta_{1}=\theta_{2}=0.7$. Inset: Shortcut level diagram for secondorder AR process across the left junction.

We have invoked higher-order AR to explain the principal step shortly behind $6 E_{c}$ in Fig. $7(\mathrm{c})$ as well as the small currents at low voltages in Figs. 7(b) and 7(c). We have not clearly associated edges to particular MARs there. Having in mind the behavior of the single superconducting junction, ${ }^{13,27}$ steps from MARs, starting from island state $n$ $=0$, could be expected at all fractions of $4 \Delta$ for the double junction. However, with the additional conditions for supplying the charging energy and decharging the island for a stationary current flow, the onsets of higher-order processes are harder to distinguish here. To construct an example where a step from second-order MARs can be found (Fig. 13), consider the following arguments. We have already derived the condition for overcoming the superconductor gap above, namely, $V>4 \Delta / 3$. Simultaneously, to charge the island up to $n=1$ by partial MARs, $V>2 E_{c}$ is required. Furthermore, to allow decharging to the right by single-charge transfer for a low transmission junction we further need $V>4 \Delta-4 E_{c}$. To unambiguously identify the onset of the process, the MAR condition should be the dominating one. Writing $E_{c}=a \Delta$ with $a$ being a real number, $4 \Delta / 3>2 E_{c}$ is equivalent to $a$ $<2 / 3$, whereas $4 \Delta / 3>4 \Delta-4 E_{c}$ means $a>2 / 3$. This proves that with parameters as in Fig. 7 a pronounced edge at $\mathrm{eV}$ $=4 \Delta / 3$ will never be found whatever the ratio between $\Delta$ and $E_{c}$. For the example in Fig. 13 we chose rather high transmissions for both junctions, such that the rate for decharging the island from 1 to 0 across the right junction by a partial AR does not become too small. First-order AR for decharging requires $V>2 \Delta-4 E_{c}$. Now, $4 \Delta / 3>2 E_{c}$ and $4 \Delta / 3>2 \Delta-4 E_{c}$ leaves a window $1 / 6<a<2 / 3 . E_{c}$ is within this range in Fig. 13 and an edge at $4 \Delta / 3$ from partial second-order MARs as well as even an edge at $4 \Delta / 4$ from partial third-order MARs are observed. $4 \Delta / 5$ falls below $2 E_{c}$, however. Without the charging-energy condition and the decharging threshold interfering too closely on the energy scale with the onsets of MAR, the $I-V$ curve resembles the single-junction characteristic with positive second-order derivative (concave instead of straight or convex form) in each step. 


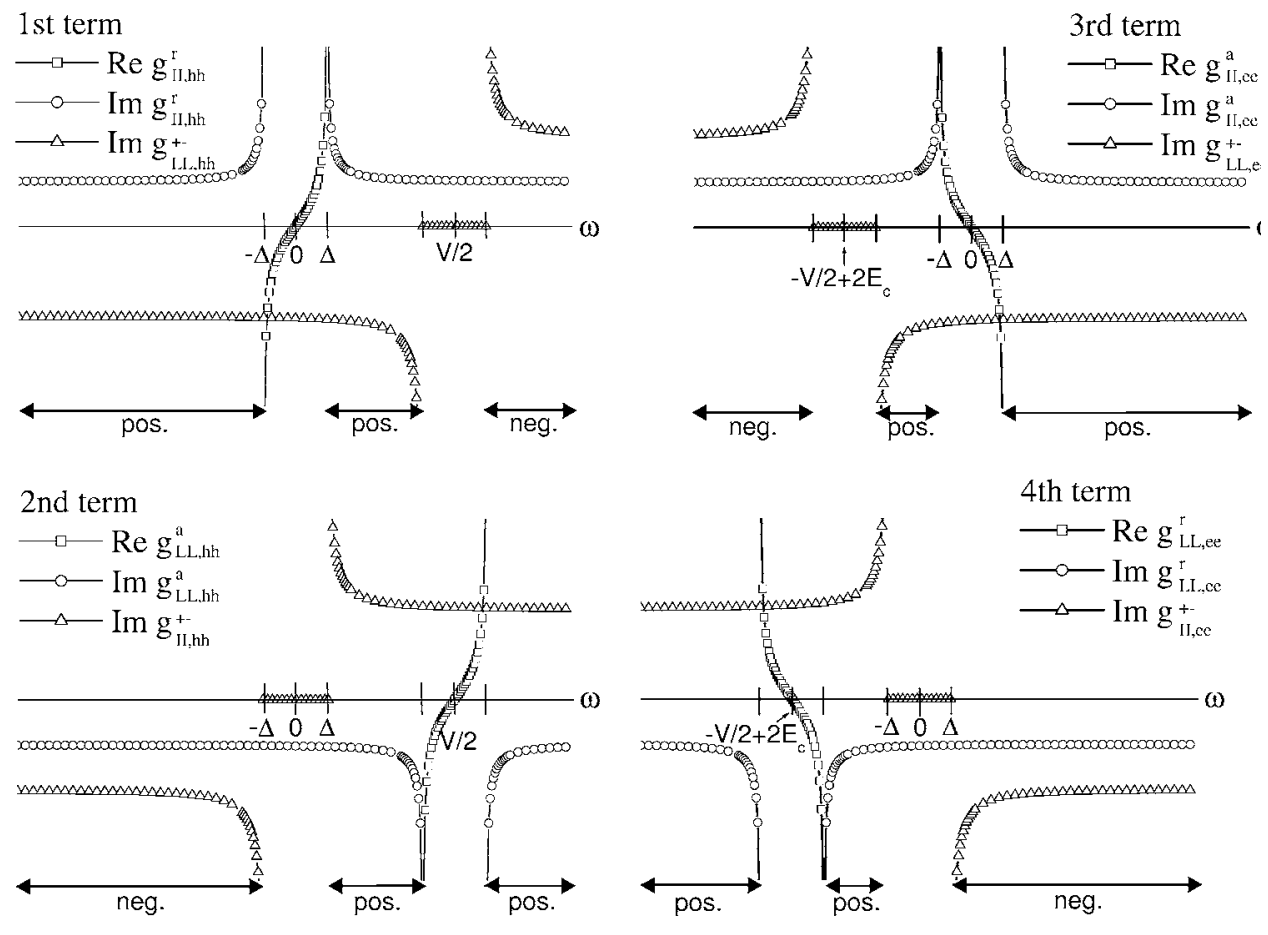

FIG. 14. Composition of the single-charge transfer lowestorder rate contributions concerning the island charge change from zero to one across the left junction for $C_{1}=C_{2}$ and $U=0$, i.e., potential of the neutral island vs the left lead $A=V / 2$. Arrows mark the sign of the product of the $g$ functions in the frequency ranges where it is real. The product is imaginary where nothing is indicated.

\section{ANALYSIS}

In this section we shall deduce how the process thresholds obtained graphically from the energy-level diagrams in the previous section arise from the rate terms (2.16) and how these formulas can lead to the selection of the higher of two thresholds associated to overcoming the superconductor gap and supplying the charging energy, respectively. The virtue of the Green's functions method lies in solving Eq. (2.3) in a closed form

$$
T=(\mathbf{1}-\sigma g)^{-1} \sigma
$$

which includes interaction to all orders and makes it valid for $\sigma$ of arbitrary size. For insight and physical interpretation it is, however, helpful to develop Eq. (4.1) into a "geometric" series, although this is not necessarily quickly converging.

$$
T=\sigma+\sigma g \sigma+\sigma g \sigma g \sigma+\ldots
$$

Expressing all $T$ in Eq. (2.16) by the sums from Eq. (4.2), the lowest-order terms will result if there is just one $\sigma$ in the place of each $T$. Then we must have $|k|=1$, because single hoppings $\sigma$ can only alter the island charge by one, and furthermore $k=-1$ in the first two lines and $k=1$ in the last two lines, for otherwise the outer indices in electron-hole space could not be $h$ or $e$, respectively. For this special case the four terms read, with $m$ evaluated from Eq. (B4):
(1) $\int d \omega g_{I I, h h}^{r}(\omega) \sigma_{I L, h}^{n n+1} g_{L L, h h}^{+-}\left(\omega-\frac{A}{\hbar}+n \frac{B}{\hbar}\right) \sigma_{L I, h}^{n+1} n$,

(2) $\int d \omega \sigma_{L I, h}^{n+1}{ }^{n} g_{I I, h h}^{+-}(\omega) \sigma_{I L, h}^{n n+1} g_{L L, h h}^{a}\left(\omega-\frac{A}{\hbar}+n \frac{B}{\hbar}\right)$,

(3) $\int d \omega \sigma_{I L, e}^{n+1}{ }^{n} g_{L L, e e}^{+-}\left(\omega+\frac{A}{\hbar}-(n+1) \frac{B}{\hbar}\right) \sigma_{L I, e}^{n n+1} g_{I I, e e}^{a}(\omega)$,

(4) $\int d \omega g_{L L, e e}^{r}\left(\omega+\frac{A}{\hbar}-(n+1) \frac{B}{\hbar}\right) \sigma_{L I, e}^{n n+1} g_{I I, e e}^{+-}(\omega) \sigma_{I L, e}^{n+1} n$.

As shown in Appendix B, frequency arguments are chosen such that all exponential phase factors cancel, including those from the $\sigma$ here; the two $\sigma$ in each of the four products thus simply give a real factor $t^{2}$. For $n=0$ the remaining $g$ functions are plotted in Fig. 14. The sign of their product is indicated in the frequency intervals where it is real, because for the rate contribution twice the real part has to be taken. The plotted functions correspond to the first two products from Eq. (2.15); the imaginary parts will be cancelled by the last two products, which are the complex conjugates.

$$
g^{r / a}(\omega)=\frac{1}{\sqrt{\Delta^{2}-(\omega \pm i \eta)^{2}}}\left(\begin{array}{cc}
-\omega \mp i \eta & \Delta \\
-\Delta & \omega \pm i \eta
\end{array}\right)
$$

$\eta$ in Eq. (4.4) is a tiny imaginary addition to the frequency for numerical performance, especially choosing the correct root. In the limit $\eta \longrightarrow 0, g$ for a fixed $\omega$ is either purely real or purely imaginary. $g^{+-}$is imaginary or zero. A product of two $g$ functions therefore is either real or purely imaginary. As 
retarded and advanced $g$ are the same function but for complex conjugation, it is easily seen that contributions to the integrals from $\omega<-\Delta$ and $\omega>V / 2+\Delta$ cancel each other for the first two terms and so do contributions for $\omega<-V / 2$ $+2 E_{c}-\Delta$ and $\omega>\Delta$ for the last two terms. The latter fact also justifies taking finite integration boundaries in the calculation. From Fig. 14 we further see that for the first two terms the remaining piece of the frequency axis between the two $2 \Delta$ intervals, where the real part of the product does not vanish, only exists if $V / 2-\Delta>\Delta$ or $V>4 \Delta$ (assuming positive $V$ here). As drawn in Fig. 14 the contribution from the interval $-V / 2+2 E_{c}+\Delta<\omega<-\Delta$ is also positive if it exists for large enough $V$, which even requires $V>4 \Delta+4 E_{c}$. Even for positive $V$, however, $-V / 2+2 E_{c}$ can come to lie on the positive $\omega$ axis, and consequently because of the sign change in $g^{+-}$the contribution will be negative. Because of the unique shape of the $g$ functions it will cancel the first two terms exactly when the intervals become of equal length, that is for $-V / 2+2 E_{c}=V / 2$ or $V=2 E_{c}$. In conclusion, the four terms thus yield a positive rate, meaning single-particle transfer across the left junction raising the island charge from zero to one, if $V>4 \Delta$ and $V>2 E_{c}$. Otherwise the rate is zero or negative, the latter meaning that lowering the island charge from one to zero across the left junction is possible. In contrast to the general series (4.2), $T_{L I}$ or $T_{I L}$ only have terms with an odd number of $\sigma$ in them, so the next higher-order rate contributions from Eq. (2.16) have one of the $T$ in each line developed into a product of the form $\sigma g \sigma g \sigma$ and the other taken as a single $\sigma$. The cases where each line contains either only $g_{e e}$ or only $g_{h h}$, that is no AR, describe multiple reflections across one junction of an electron or a hole and yield the same voltage thresholds as the single-particle terms from Eq. (4.3) in accordance with density-of-states and charging-energy requirements. To discuss the simple case of vanishing gate voltage and equal junction capacitances, the rate contribution from $n=0$ to 1 for positive $V$ only exists if $V>4 \Delta$ and changes sign at $V=2 E_{c}$. It comes out negative for higher voltage, which is correct though, because the rate contribution here is linked to the second term of the series that we get when developing the square root of the transmission probability $\theta$ in terms of the hopping amplitude $t$

$$
\sqrt{\theta}=\frac{2 t}{1+t^{2}}=2\left(t-t^{3}+\ldots\right) .
$$

The number of hopping amplitude factors in each contribution in

$$
\begin{aligned}
g T g T & =g(\sigma+\sigma g \sigma g \sigma+\ldots) g(\sigma+\sigma g \sigma g \sigma+\ldots) \\
& =g \sigma g \sigma+g \sigma g \sigma g \sigma g \sigma+\ldots
\end{aligned}
$$

can be mapped onto the powers of $t$ in the composition of the transmission probability

$$
\theta=\sqrt{\theta} \sqrt{\theta}=4\left(t^{2}-2 t^{4}+\ldots\right) .
$$

Again for the special case of $U=0$ and $C_{1}=C_{2}$, for the right junction instead of the left, $V / 2$ [from Figs. 14(a) and 14(b)] would have to be replaced by $-V / 2$, and $-V / 2+2 E_{c}$ [from Figs. $14(\mathrm{c})$ and $14(\mathrm{~d})]$ by $V / 2+2 E_{c}$, still regarding island charge changes between zero and one. The $2 \Delta$ interval around $V / 2+2 E_{c}$ is then separated from the $2 \Delta$ interval around $\omega=0$ if $V / 2+2 E_{c}-\Delta>\Delta$ or $V>4 \Delta-4 E_{c}$. All four terms will give a negative rate then meaning that decharging the island from one to zero across the right junction is possible. There is no counterbalancing of the first two against the last two terms for positive voltages, as $V / 2+2 E_{c}$ cannot equal $-V / 2$ for $V>0$. Thus $V>4 \Delta-4 E_{c}$ is the unique threshold for decharging. Our analysis has thus so far explained the thresholds due to single-particle transfer found in the $I-V$ curves from the last section. To complete the given information, we note that in the general case with unequal junction capacitances and/or nonvanishing gate voltage the condition to increase the island charge from $n$ to $n+1$ by single-particle transfer across the left junction is that $2 A$ has to exceed the higher value out of $4 \Delta+4 n E_{c}$ and $(4 n+2) E_{c}$. For decharging from $n+1$ to $n$ by single-particle transfer across the right junction, it is required that $-2 C>4 \Delta-4(n$ $+1) E_{c}$.

A similar rate-term study as for the single-particle transfers shall now be outlined for the lowest-order AR. Inserting the developments of all $T$ in (2.16), from all terms that consist of four $\sigma$ and have $g_{e h}$ and $g_{h e}$ in them, we pick a group of four for illustration (there are sixteen such products on the whole). As Ansatz to derive the frequency arguments of the two $g$ in each product that are not yet explicitly written in Eq. (2.16) use that all exponential phase factors have to cancel in these dc current rates. Apart from an amplitude factor of $t^{4}$ the following products remain for integration over $\omega$.

The first term with $k=1\left(g_{I I}^{r} T_{I L}^{n n-1} g_{L L}^{+-} T_{L I}^{n-1}{ }^{n}\right)_{h h}$ contains, developing the second $T$,

$$
g_{I I, h e}^{r} \sigma_{I L, e}^{n n-1} g_{L L, e e}^{+-} \sigma_{L I, e}^{n-1} g_{I I, e h}^{a} \sigma_{I L, h}^{n n+1} g_{L L, h h}^{a} \sigma_{L I, h}^{n+1 ~ n}
$$

which reduces to

$$
\begin{aligned}
& \text { (I) } g_{I I, h e}^{r}(\omega) g_{L L, e e}^{+-}\left(\omega+\frac{A}{\hbar}-n \frac{B}{\hbar}\right) g_{I I, e h}^{a}(\omega) \\
& \times g_{L L, h h}^{a}\left(\omega-\frac{A}{\hbar}+n \frac{B}{\hbar}\right)
\end{aligned}
$$

and with $k=-1$ in $\left(g_{I I}^{r} T_{I L}^{n{ }^{n+1}} g_{L L}^{+-} T_{L I}^{n+1}{ }^{n}\right)_{h h}$ there is, developing the first $T$,

$$
g_{I I, h e}^{r} \sigma_{I L, e}^{n n-1} g_{L L, e e}^{r} \sigma_{L I, e}^{n-1}{ }^{n} g_{I I, e h}^{r} \sigma_{I L, h}^{n n+1} g_{L L, h h}^{+-} \sigma_{L I, h}^{n+1 ~ n}
$$

which gives

$$
\begin{aligned}
& \text { (II) } g_{I I, h e}^{r}(\omega) g_{L L, e e}^{r}\left(\omega+\frac{A}{\hbar}-n \frac{B}{\hbar}\right) g_{I I, e h}^{r}(\omega) \\
& \times g_{L L, h h}^{+-}\left(\omega-\frac{A}{\hbar}+n \frac{B}{\hbar}\right)
\end{aligned}
$$

The third term $\left(T_{I L}^{n+1}{ }^{n+2} g_{L L}^{+-} T_{L I}^{n+2}{ }^{n+1} g_{I I}^{a}\right)_{e e}$ for $k=-1$ developing the first $T$ has in it

$$
\sigma_{I L, e}^{n+1}{ }^{n} g_{L L, e e}^{r} \sigma_{L I, e}^{n+1} g_{I I, e h}^{r} \sigma_{I L, h}^{n+1}{ }^{n+2} g_{L L, h h}^{+-} \sigma_{L I, h}^{n+2}{ }^{n+1} g_{I I, h e}^{a}
$$

or 


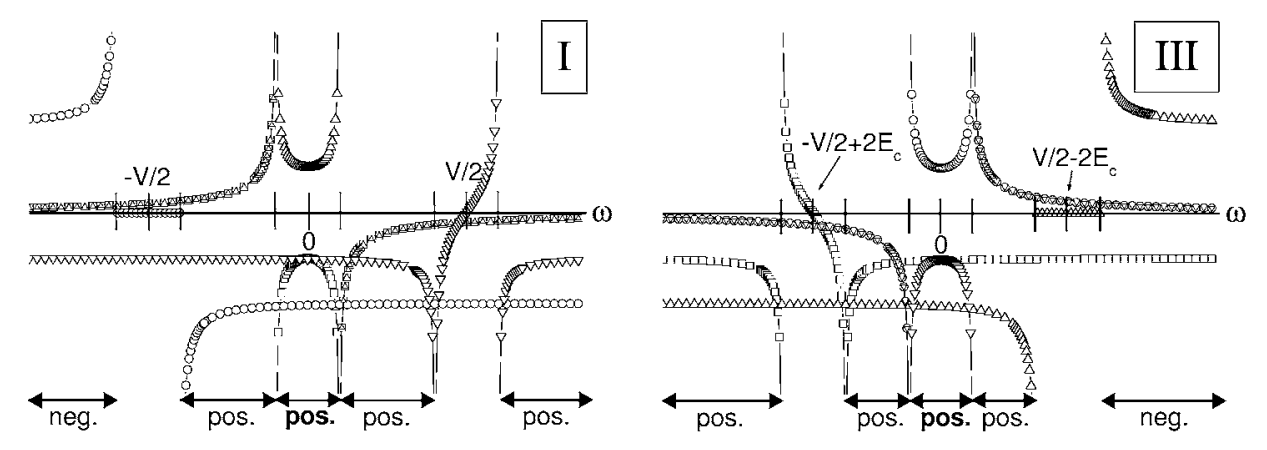

FIG. 15. Composition of lowest-in-voltage contributions from first-order AR to islandcharge change rates from zero to one across the left junction for potential difference $A=V / 2$. $g$ functions for terms I-IV (see text) are plotted with the same symbols for their purely real values within the intervals of lengths $2 \Delta$ and their purely imaginary values outside these. Arrows mark the sign of the product where it is real.

(III)

$$
g_{L L, e e}^{r}\left(\omega+\frac{A}{\hbar}-(n+1) \frac{B}{\hbar}\right) g_{I I, e h}^{r}(\omega)
$$$$
\times g_{L L, h h}^{+-}\left(\omega-\frac{A}{\hbar}+(n+1) \frac{B}{\hbar}\right) g_{I I, h e}^{a}(\omega)
$$

and with $k=1$, in $\left(T_{I L}^{n+1}{ }^{n} g_{L L}^{+-} T_{L I}^{n n+1} g_{I I}^{a}\right)_{e e}$ developing the second $T$, one contribution is

$$
\sigma_{I L, e}^{n+1}{ }^{n} g_{L L, e e}^{+-} \sigma_{L I, e}^{n n+1} g_{I I, e h}^{a} \sigma_{I L, h}^{n+1{ }^{n+2}} g_{L L, h h}^{a} \sigma_{L I, h}^{n+2}{ }^{n+1} g_{I I, h e}^{a}
$$

equal to

$$
\begin{aligned}
& \text { (IV) } g_{L L, e e}^{+-}\left(\omega+\frac{A}{\hbar}-(n+1) \frac{B}{\hbar}\right) g_{I I, e h}^{a}(\omega) \\
& \times g_{L L, h h}^{a}\left(\omega-\frac{A}{\hbar}+(n+1) \frac{B}{\hbar}\right) g_{I I, h e}^{a}(\omega) .
\end{aligned}
$$

The $g$ functions appearing in the four products (4.8) are plotted in Fig. 15 for the same special case as Fig. 14, that is, take $n=0$ and $A=V / 2$ in Eq. (4.8). Here, in the central interval of length $2 \Delta$ around zero there are two real and two imaginary $g$, so the product is real. For symmetry reasons all contributions from outside the central $2 \Delta$ intervals cancel. Contributions from the finite intervals between the three $2 \Delta$ regions cancel between terms I and II and between III and IV, respectively. Contributions from the outermost half-open intervals already cancel in each of the terms I to IV alone. It is the contribution from the central $2 \Delta$-part, meaning the energy range inside the gap on the island, that counts for AR. This contribution is positive in all four terms as they are drawn in Fig. 15. To avoid that the other two $2 \Delta$ intervals completely overlap the central one and thus leave no frequency range with real and nonzero product there, $V / 2-\Delta$ $>0$ or $V>2 \Delta$ is required in terms I and II, whereas in terms III and IV one needs $V / 2-2 E_{c}-\Delta>0$ or $V>2 \Delta+4 E_{c}$. Even restricting our considerations to $V>0,-V / 2+2 E_{c}$ and $V / 2$ $-2 E_{c}$ with their $2 \Delta$ intervals around them could swap sides in terms III and IV reversing the sign of the product in the range between them. If lengths of intervals become equal to those from terms I and II, terms III and IV can outweight them, and this is the case for $-V / 2=V / 2-2 E_{c}$ or $V=2 E_{c}$, where the rate contribution consequently changes sign (if it is not still zero in case that $2 \Delta$ is the greater threshold). For the lowest-order AR again our analysis proves that the threshold values have been given correctly in the previous section. In general, the conditions to increase the island charge from $n$ to $n+1$ by partial lowest-order AR across the left junction read $2 A>2 \Delta+4 n E_{c}$ and $2 A>(4 n+2) E_{c}$. All other terms with four $g$ containing $g_{e h}$ and $g_{h e}$ for AR, can similarly be taken in groups of four and shown to cancel completely or give their essential rate contribution from a $2 \Delta$ interval. However, not all half-open outer and intermediate intervals fully outweight each other for all voltages and arbitrary ratio $E_{c}: \Delta$. Their contributions are strongly damped because $g_{e h}$ and $g_{h e}$ quickly drop to almost zero outside the $2 \Delta$ intervals where they are centered, but they may already be seen as an effect of interference between island charge states. An essential contribution from AR with the electronhole conversion in the left lead instead of on the islandeven if only partially carried out for island charginginterestingly requires the full charging energy for both particles, that is $4 E_{c}$ for the process from island charge -1 to 1 and $8 E_{c}$ for going from 0 to 2 . This behavior adds further small steps or changes in slope to the $I-V$ at these voltage positions.

The products in Eq. (4.8) represent contributions to the rate for changing the island charge between $n$ and $n+1$. A $\sigma$ directly at the beginning or at the end of a product always designates a hopping between $n$ and $n+1$. Charge indices $n-1$ and $n+2$ and thus densities of states corresponding to these island potentials also appear. In products containing 


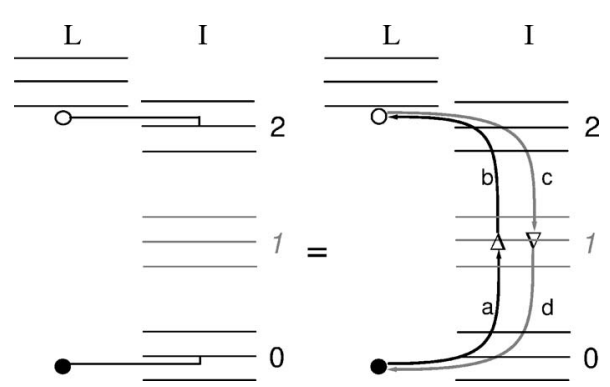

FIG. 16. The AR bringing the island from state 0 to 2 can equivalently be drawn as mirroring the level of the incoming electron at the intermediate one state. Adding the complementary path of the process corresponds to evaluating the intensity from the amplitude. Arrows and points are identified with the factors of terms III and IV from Eq. (4.8) with $n=0$ as follows: $(\bullet) g_{L L, e e}$, (a) $\sigma_{L I, e}^{01}$,

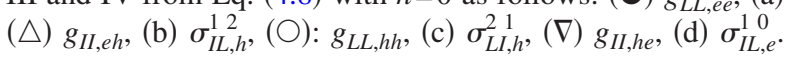

even more factors $\sigma$, the island charge index may adopt values even further away from $n$ and $n+1$. So the explicit expressions from Eq. (4.8) again as in Fig. 3(b) illustrate how interference between island charge states is included despite using rates between neighboring charge states only. Developments such as Eq. (4.8) can be regarded as interferences between transport processes of different order. For example, the product behind $g^{+-}$in term IV with $n=0$ corresponds to the second process from Fig. 3(b) going from 0 to 1 , from 1 to 2 , and back to 1 . Instead of the reverse of this three-step process, here the conjugate of the simple one-step process $0 \rightarrow 1$, the $\sigma$ in front of $g^{+-}$, closes the cycle to $n=0$. The more island charge states get involved in a rate contribution, the more $g$ functions shifted against each other have to be multiplied. The frequency range to integrate over will decompose into more $2 \Delta$ and intermediate intervals. With more complicated conditions in terms of $E_{c}$ and $\Delta$ there can well be more than one central part yielding a nonzero rate and thus giving rise to current contributions like the small bump at low voltages in Fig. 7(b).

We shall end this section by giving yet another interpretation of the rate terms (4.8). Terms I and II designate the AR between $n=-1$ and $n=1$, terms III and IV the AR between $n=0$ and $n=2$ with the AR on the island from Fig. 8(b), and taking the $0 \leftrightarrow 1$ step out of both here. For the second process we illustrate in Fig. 16 that the diagram from Fig. 8(b) would be equal to mirroring the energy level at the $n=1$ state. Going the AR path forth and back in the diagram corresponds to converting the amplitude for the process into an intensity. Just shifting the leading $\sigma$ to the end of the terms III and IV (see Appendix B), the elements of the path are easily identified with the factors of the rate products.

\section{CONCLUSIONS}

In conclusion, we have developed a model to calculate current-voltage curves $(I-V \mathrm{~s})$ for a series of two quantum point contacts (QPCs) with a bulklike metallic island between them in the superconducting state. Charge transfer processes over both junctions can be simultaneous and are linked via the varying island potential. Coherence is, how- ever, restricted to multiple reflections across individual junctions. Nonlinearities or edges in the $I-V \mathrm{~s}$ are due to an interplay of multiple Andreev reflections (MARs) and Coulomb blockade (CB). Our model predicts that MARs do not principally get suppressed because of CB. Our method is based on coupling amplitudes from single-hopping events rather than addition of current contributions from cotunneling or multiple reflections. Features in the $I-V \mathrm{~s}$ are nevertheless conveniently interpreted as arising from processes of particular order. Interestingly, instead of only one threshold for a certain charge transfer, we get two, one assuring to overcome the superconductor energy gap and the other checking for the charging energy. In contrast to orthodox theory (OT) (without cotunneling), even a current contribution from a MAR only requires the energy for one additional charge on the island. The ratio of the gap width and the charging energy then determines which is the higher threshold marking the onset of the process. Flow of a dc current through the whole system further requires fulfilling such conditions for a process increasing the island charge across one junction and at the same time for a process decreasing the island charge across the other junction. Therefore there is no unique sequence of step positions in the $I-V$, but the shape of the curve depends crucially on the setup parameters.

Extension of the model to include coherent forth- and back-reflections over both junctions is possible and currently under work. This fully coherent model produces $I-V$ characteristics that differ from OT even in the normal-conducting state, with earlier current onset, however CB effects still present. Other modifications to consider would consist in allowing the leads and the island to be of different materials with nonequal gap widths or even regarding superconductornormal conductor mixed systems, as well as including a parity effect ${ }^{28-30}$ possibly by according the density of states for an odd number of excess charges on the island. Our model for double-QPCs even in the low transmission regime predicts a different behavior than that observed in experiments on and extended orthodox theory calculations for double tunnel junctions. ${ }^{20,24}$ For a QPC and a tunnel junction in series we can therefore also propose a hybrid model promising to reproduce qualitative features from preliminary measurements ${ }^{17}$ with transport channels in the intermediate transmission regime for the QPC.

\section{ACKNOWLEDGMENTS}

We thank the Deutsche Forschungsgemeinschaft and the Landesstiftung Baden-Württemberg for financial support.

\section{APPENDIX A}

In this section we show the blockwise written Dyson equation $T=\sigma+\sigma g T$ for processes across the left junction in the time domain 


$$
\begin{aligned}
& \left(\begin{array}{llll}
T_{L L, e e}^{n_{1} n_{2}} & T_{L L, e h}^{n_{1} n_{2}} & T_{L, n_{1} e}^{n_{1} n_{2}} & T_{L I,, h}^{n_{1} n_{2}} \\
T_{L L, h e}^{n_{1} n_{2}} & T_{L L, h h}^{n_{1} n_{2}} & T_{L l, h e}^{n_{1} n_{2}} & T_{L I, h h}^{n_{1} n_{2}} \\
T_{I L, e e}^{n_{1} n_{2}} & T_{I L, e h}^{n_{1} n_{2}} & T_{I I, e e}^{n_{1} n_{2}} & T_{I I, e h}^{n_{1} n_{2}} \\
T_{I L, h e}^{n_{1} n_{2}} & T_{I L, h h}^{n_{1} n_{2}} & T_{I I, h e}^{n_{1} n_{2}} & T_{I I, h h}^{n_{1} n_{2}}
\end{array}\right) \\
& =(\delta_{n_{1}+1, n_{2}} \underbrace{\left(\begin{array}{cccc}
0 & 0 & t e^{i \tau\left(-A+\left(n_{1}+1\right) B\right) / \hbar} & 0 \\
0 & 0 & 0 & 0 \\
0 & 0 & 0 & 0 \\
0-t e^{i \tau\left(-A+n_{1} B\right) / \hbar} & 0 & 0
\end{array}\right)}_{\sigma_{n_{1}, n_{1}+1}}+\delta_{n_{1}-1, n_{2}}^{\left(\begin{array}{cccc}
0 & 0 & 0 & 0 \\
0 & 0 & 0 & -t e^{i \tau\left(A-\left(n_{1}-1\right) B\right) / \hbar} \\
t e^{i \tau\left(A-n_{1} B\right) / \hbar} & 0 & 0 & 0 \\
0 & 0 & 0 & 0
\end{array}\right)} \underbrace{\left(\begin{array}{cc}
0 \\
0
\end{array}\right)}_{\sigma_{n_{1}, n_{1}-1}}
\end{aligned}
$$

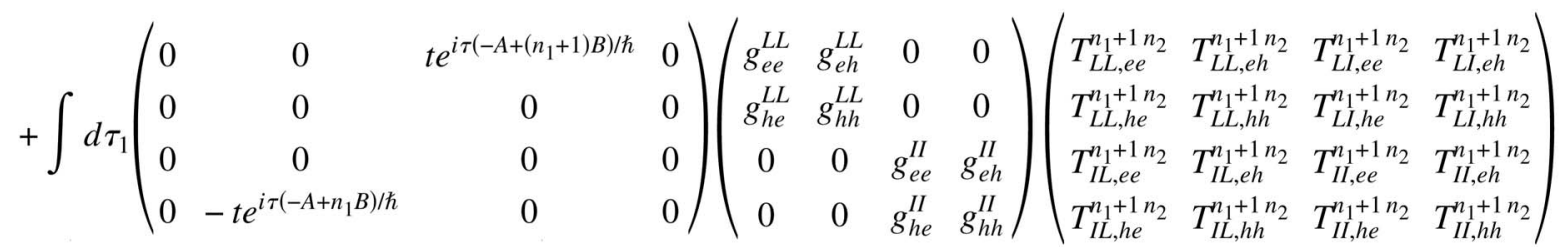

$$
\begin{aligned}
& +\int d \tau_{1}\left(\begin{array}{cccc}
0 & 0 & 0 & 0 \\
0 & 0 & 0 & -t e^{i \tau\left(A-\left(n_{1}-1\right) B\right) / \hbar} \\
t e^{i \tau\left(A-n_{1} B\right) / \hbar} & 0 & 0 & 0 \\
0 & 0 & 0 & 0
\end{array}\right)\left(\begin{array}{cccc}
g_{e e}^{L L} & g_{e h}^{L L} & 0 & 0 \\
g_{h e}^{L L} & g_{h h}^{L L} & 0 & 0 \\
0 & 0 & g_{e e}^{I I} & g_{e h}^{I I} \\
0 & 0 & g_{h e}^{I I} & g_{h h}^{I I}
\end{array}\right)\left(\begin{array}{cccc}
T_{L L, e e}^{n_{1}-1 n_{2}} & T_{L L, e h}^{n_{1}-1 n_{2}} & T_{L I, e e}^{n_{1}-1 n_{2}} & T_{L I, e h}^{n_{1}-1 n_{2}} \\
T_{L, h e}^{n_{1}-1 n_{2}} & T_{L L, h h}^{n_{1}-1 n_{2}} & T_{L I, h e}^{n_{1}-1 n_{2}} & T_{L I, h h}^{n_{1}-1 n_{2}} \\
T_{I L, e e}^{n_{1}-1 n_{2}} & T_{I L, e h}^{n_{1}-1 n_{2}} & T_{I I, e e}^{n_{1}-1 n_{2}} & T_{I I, e h}^{n_{1}-1 n_{2}} \\
T_{I L, h e}^{n_{1}-1 n_{2}} & T_{I L, h h}^{n_{1}-1 n_{2}} & T_{I I, h e}^{n_{1}-1 n_{2}} & T_{I I, h h}^{n_{1}-1 n_{2}}
\end{array}\right)
\end{aligned}
$$

The time argument of all $g$ on the right side is $\left(\tau-\tau_{1}\right) . T$ on the left side is of $\left(\tau, \tau^{\prime}\right)$ and $T$ on the right side under the integrals is of $\left(\tau_{1}, \tau^{\prime}\right)$.

If an electron is just once transferred from $I$ to $L$ (position $\left.T_{L I, e e}\right)$ the number of (negative) charges on the island decreases by one, therefore $n_{1}=n_{2}-1$ or $n_{2}=n_{1}+1$. The (real) transfer amplitude is $t$. The fixed part due to the applied voltage $V$ of the potential difference is $A$, taken with a minus sign in the exponent for the electron's negative charge. The varying part of the island potential is $-\left(n_{1}+1\right) B$ before and $-n_{1} B$ after the transfer. As the electron moves from the island to the fixed potential on the left it is the value before that enters the phase change. The other cases are as follows. An electron goes from $L$ to $I$ (position $T_{I L, e e}$ ): The number of negative charges on the island increases by one, $n_{1}=n_{2}+1$ or $n_{2}=n_{1}-1$. The potential difference in the exponent is taken with opposite sign than in the first case, because the electron is going the other way. It is moving onto the island, thus it is the island potential afterwards, i.e., $-n_{1} B$, that matters. A hole goes from $I$ to $L$ (position $T_{L I, h)}$ ): The (negative) island charge increases by 1 , thus $n_{1}=n_{2}+1$ or $n_{2}=n_{1}-1$. Without the phase factor from the potential the hopping amplitude for the hole is $-t$. The potential difference is taken with opposite sign in the exponent than for an electron going from $I$ to $L$, because the hole has opposite charge. It is the island potential before the transfer that matters, i.e., $-\left(n_{1}-1\right) B$. A hole goes from $L$ to $I$ (position $T_{I L, h h}$ ): The (negative) island charge decreases, $n_{1}=n_{2}-1$ or $n_{2}=n_{1}+1$. The amplitude is $-t$ for the hole and we have to take the potential in the exponent with opposite sign as for an electron going from $L$ to $I$ as well as the island potential afterwards, i.e., $-n_{1} B$.

Recursion relation for $T_{L I}$ in Fourier space: 


$$
\begin{aligned}
& \left(\begin{array}{ll}
T_{k m}^{n_{1} n_{2}, e e} & T_{k m}^{n_{1} n_{2}, e h} \\
T_{k m}^{n_{1} n_{2}, h e} & T_{k m}^{n_{1} n_{2}, h h}
\end{array}\right)=\delta_{n_{1}+1, n_{2}} \delta_{k, 1} \delta_{m,-n_{1}-1} \underbrace{\left(\begin{array}{cc}
t & 0 \\
0 & 0
\end{array}\right)}_{s_{+1}}+\delta_{n_{1}-1, n_{2}} \delta_{k,-1} \delta_{m, n_{1}-1} \underbrace{\left(\begin{array}{cc}
0 & 0 \\
0 & -t
\end{array}\right)}_{s_{-1}} \\
& +t^{2}\left(\begin{array}{cc}
g_{e e}^{I I}\left(\omega+(k-1) A+\left(m+n_{1}+1\right) B\right) & g_{e e}^{I I}\left(\omega+(k-1) A+\left(m+n_{1}+1\right) B\right) \\
\times g_{e e}^{L L}(\omega+k A+m B) & \times g_{e h}^{L L}(\omega+k A+m B) \\
g_{h h}^{I I}\left(\omega+(k+1) A+\left(m-n_{1}+1\right) B\right) & g_{h h}^{I I}\left(\omega+(k+1) A+\left(m-n_{1}+1\right) B\right) \\
\times g_{h e}^{L L}(\omega+k A+m B) & \times g_{h h}^{L L}(\omega+k A+m B)
\end{array}\right) T_{k m}^{n_{1} n_{2}} \\
& \epsilon_{k} \\
& -t^{2}\left(\begin{array}{cc}
g_{e h}^{I I}\left(\omega+(k-1) A+\left(m+n_{1}+1\right) B\right) & g_{e h}^{I I}\left(\omega+(k-1) A+\left(m+n_{1}+1\right) B\right) \\
\times g_{h e}^{L L}\left(\omega+(k-2) A+\left(m+2 n_{1}+2\right) B\right) & \times g_{h h}^{L L}\left(\omega+(k-2) A+\left(m+2 n_{1}+2\right) B\right) \\
0 & 0
\end{array}\right) T_{k-2, m+2 n_{1}+2}^{n_{1}+2 n_{2}} \\
& V_{k}^{-} \\
& \underbrace{-t^{2}\left(\begin{array}{cc}
0 & 0 \\
g_{h e}^{I I}\left(\omega+(k+1) A+\left(m-n_{1}+1\right) B\right) & g_{h e}^{I I}\left(\omega+(k+1) A+\left(m-n_{1}+1\right) B\right) \\
\times g_{e e}^{L L}\left(\omega+(k+2) A+\left(m-2 n_{1}+2\right) B\right) & \times g_{e h}^{L L}\left(\omega+(k+2) A+\left(m-2 n_{1}+2\right) B\right)
\end{array}\right)}_{V_{k}^{+}} T_{k+2, m-2 n_{1}+2 .}^{n_{1}-2 n_{2}} .
\end{aligned}
$$

$T$ in Eq. (A2) always means the matrix $\left(\begin{array}{l}T_{e e} T_{e h} \\ T_{h e} T_{h h}\end{array}\right) . \epsilon_{k}, V_{k}$, and $V_{k}^{+}$include the factor $t^{2}$ and $V_{k}^{-}$and $V_{k}^{+}$also the minus sign in front of it, see Eq. (2.11).

In order to solve it, we define $z_{k}^{+}$and $z_{k}^{-}$by

$$
T_{k+2}=z_{k}^{+} T_{k} \quad \text { and } \quad T_{k-2}=z_{k}^{-} T_{k}
$$

from which follows that

$$
z_{k-2}^{+}=\left(\mathbf{1}-\epsilon_{k}-V_{k}^{+} z_{k}^{+}\right)^{-1} V_{k} \quad \text { for } k>1
$$

and

$$
z_{k+2}^{-}=\left(\mathbf{1}-\epsilon_{k}-V_{k}^{-} z_{k}^{-}\right)^{-1} V_{k}^{+} \quad \text { for } k<-1 .
$$

The $z_{k}^{ \pm}$are calculated beginning at some $k_{\max }-2$ or $k_{\min }+2$, respectively, for which $V_{k_{\max }}^{+}$or $V_{k_{\min }}$ are assumed to vanish, for they would lead to $T_{k_{\max }+2}$ or $T_{k_{\min }-2}$, respectively, which are not included any more. $T_{1}$ and $T_{-1}$ are obtained from

$$
\begin{gathered}
T_{1}=s_{1}+\epsilon_{1} T_{1}+V_{1}^{+} z_{1}^{+} T_{1}+V_{1}^{-} T_{-1}, \\
T_{-1}=s_{-1}+\epsilon_{-1} T_{-1}+V_{-1}^{+} T_{1}+V_{-1}^{-} z_{-1}^{-} T_{-1} .
\end{gathered}
$$

Then $T_{k}$ for positive $k$ can be successively evaluated using $z_{k}^{+}$ and $T_{k}$ for negative $k$ using $z_{k}^{-}$.

\section{APPENDIX B}

Here we demonstrate how the rates given by the Keldysh Green's functions are rewritten in terms of the transfer functions and how the transformation from time to Fourier space is performed. Noting both island charge indices on a $\sigma$ yields equivalent information to giving one of them and designating the $\sigma$ as an $e$ or $h$ transfer. As we take the trace in $e h$ space of Eq. (2.15), an outermost $\sigma$ can be moved from the beginning to the end of a matrix product or from the end to the beginning; this changes the outer charge index from $n$ to $n+1$ or vice versa. For the same reason, deciding that we take the $h h$ component of the first and the ee component of the second product, the inner charge index can as well be understood as being summed over, as are all of them in the following. For simplicity we will, however, cease to write them explicitly. 


$$
\begin{aligned}
& \operatorname{Tr}\left(\sigma_{L I}^{n+1 n} G_{I L,+-}^{n n+1}+G_{L I,+-}^{n n+1} \sigma_{I L}^{n+1 ~ n}\right)=\sum_{n^{\prime}}\left(\sigma_{L I}^{n+1 n^{\prime}} G_{I L,+-}^{n^{\prime} n+1}\right)_{h h}+\sum_{n^{\prime}}\left(G_{L I,+-}^{n n^{\prime}} \sigma_{I L}^{n^{\prime}{ }^{n}}\right)_{e e} \\
& =\left[\sigma_{L I}\left(G_{I I}^{r} \sigma_{I L} g_{L L}^{+-}\left(1+\sigma_{L I} G_{I L}^{a}\right)+\sigma_{L I}\left(\mathbf{1}+G_{I L}^{r} \sigma_{L I}\right) g_{I I}^{+-} \sigma_{I L} G_{L L}^{a}\right)\right]_{h h}^{\text {outer index } n+1} \\
& +\left[\left(\mathbf{1}+G_{L I}^{r} \sigma_{I L}\right) g_{L L}^{+-} \sigma_{L I} G_{I I}^{a} \sigma_{I L}+G_{L L}^{r} \sigma_{L I} g_{I I}^{+-}\left(\mathbf{1}+\sigma_{I L} G_{L I}^{a}\right) \sigma_{I L}\right]_{e e}^{\text {outer index } n} \\
& =\left[G_{I I}^{r} \sigma_{I L} g_{L L}^{+-}\left(\sigma_{L I}+\sigma_{L I} G_{I L}^{a} \sigma_{L I}\right)\right]_{h h}^{n}+\left[\left(\sigma_{L I}+\sigma_{L I} G_{I L}^{r} \sigma_{L I}\right) g_{I I}^{+-} \sigma_{I L} G_{L L}^{a}\right]_{h h}^{n+1} \\
& +\left[\left(\sigma_{I L}+\sigma_{I L} G_{L I}^{r} \sigma_{I L}\right) g_{L L}^{+-} \sigma_{L I} G_{I I}^{a}\right]_{e e}^{n+1}+\left[G_{L L}^{r} \sigma_{L I} g_{I I}^{+-}\left(\sigma_{I L}+\sigma_{I L} G_{L I}^{a} \sigma_{I L}\right)\right]_{e e}^{n} \\
& =\left[g_{I I}^{r} T_{I L}^{r} g_{L L}^{+-}\left(\sigma_{L I}+\sigma_{L I} g_{I I}^{a} T_{I I}^{a}\right)\right]_{h h}^{n}+\left[\left(\sigma_{L I}+\sigma_{L I} g_{I I}^{r} T_{I I}^{r}\right) g_{I I}^{+-} T_{I L}^{a} g_{L L}^{a}\right]_{h h}^{n+1} \\
& +\left[\left(\sigma_{I L}+\sigma_{I L} g_{L L}^{r} T_{L L}^{r}\right) g_{L L}^{+-} T_{L I}^{a} g_{I I}^{a}\right]_{e e}^{n+1}+\left[g_{L L}^{r} T_{L I}^{r} g_{I I}^{+-}\left(\sigma_{I L}+\sigma_{I L} g_{L L}^{a} T_{L L}^{a}\right)\right]_{e e}^{n} \\
& =\left[g_{I I}^{r} T_{I L}^{r} g_{L L}^{+-} T_{L I}^{a}\right]_{h h}^{n}+\left[T_{L I}^{r} g_{I I}^{+-} T_{I L}^{a} g_{L L}^{a}\right]_{h h}^{n+1}+\left[T_{I L}^{r} g_{L L}^{+-} T_{L I}^{a} g_{I I}^{a}\right]_{e e}^{n+1}+\left[g_{L L}^{r} T_{L I}^{r} g_{I I}^{+-} T_{I L}^{a}\right]_{e e}^{n},
\end{aligned}
$$

where Eq. (2.3) has been used in the last step. Complementary to Eq. (2.10), for $T_{I L}$ set the Fourier transform

$$
T_{I L}^{n_{1} n_{2}}\left(\tau, \tau^{\prime}\right)=\sum_{k} \sum_{m} \int d \omega e^{-i \omega \tau} e^{i k A \tau^{\prime} / \hbar} e^{i m B \tau^{\prime} / \hbar} e^{i \omega \tau^{\prime}} T_{I L, k m}^{n_{1} n_{2}}(\omega)
$$

with $k=n_{1}-n_{2}$ and $m=-k n_{2}-k^{2} / 2-1 / 2$. Then for the function in Fourier space we can exploit (an asterisk denotes complex conjugation)

$$
\begin{gathered}
\left(T_{L I, e e}^{n_{1} n_{2}, r / a}\right)^{*}=T_{I L, e e}^{n_{2} n_{1}, a / r}, \\
\left(T_{L I, e h}^{n_{1} n_{2}, r / a}\right)^{*}=-T_{I L, h e}^{n_{2} n_{1}, a / r}, \\
\left(T_{L I, h e}^{n_{1} n_{2}, r / a}\right)^{*}=-T_{I L, e h}^{n_{2} n_{1}, a / r}, \\
\left(T_{L I, h h}^{n_{1} n_{2}, r / a}\right)^{*}=T_{I L, h h}^{n_{2} n_{1}, a / r} .
\end{gathered}
$$

It still has to be proven that in building a product with Eq. (2.10) for $T_{L I}$ and Eq. (B2) for $T_{I L}$ only contributions with the same $k$ from both sums remain and that in Eq. (2.16) in each product $T_{L I}, T_{I L}$, and $g_{I I}$ have the same argument and the argument of $g_{L L}$ is shifted by $k A+m B$. In Eq. (2.16) $m$ is the index of both $T$ from each product, i.e., in the order the terms are written

$$
\begin{gathered}
m=-k(n-k)-k^{2} / 2-1 / 2, \\
m=-k(n+1)-k^{2} / 2-1 / 2, \\
m=-k(n+1-k)-k^{2} / 2-1 / 2, \\
m=-k n-k^{2} / 2-1 / 2 .
\end{gathered}
$$

As an example for the way to proceed, we here take the first out of the four terms (still in the time domain) and insert the Fourier representations (2.9), (2.10), and (B2):

$$
\begin{aligned}
{\left[g_{I I}^{r} T_{I L}^{r} g_{L L}^{+-} T_{L I}^{a}\right]_{h h}^{n}\left(\tau, \tau^{\prime}\right)=} & \sum_{n^{\prime}} \int d \tau_{1} d \tau_{2} d \tau_{3} g_{I I}^{r}\left(\tau-\tau_{1}\right) T_{I L}^{n n^{\prime}, r}\left(\tau_{1}, \tau_{2}\right) g_{L L}^{+-}\left(\tau_{2}-\tau_{3}\right) T_{L I}^{n^{\prime} n, a}\left(\tau_{3}, \tau^{\prime}\right) \\
= & \sum_{n^{\prime}} \int d \tau_{1} d \tau_{2} d \tau_{3} \sum_{k} \sum_{m} \sum_{k^{\prime}} \sum_{m^{\prime}} \int d \omega_{1} d \omega_{2} d \omega_{3} d \omega_{4} e^{-i \omega_{1}\left(\tau-\tau_{1}\right)} g_{I I}^{r}\left(\omega_{1}\right) e^{-i \omega_{2} \tau_{1}} e^{i k A \tau_{2}} e^{i m B \tau_{2}} e^{i \omega_{2} \tau_{2}} \\
& \times T_{I L, k m}^{n n^{\prime}, r}\left(\omega_{2}\right) e^{-i \omega_{3}\left(\tau_{2}-\tau_{3}\right)} g_{L L}^{+-}\left(\omega_{3}\right) e^{-i \omega_{4} \tau_{3}} e^{-i k^{\prime} A \tau_{3}} e^{-i m^{\prime} B \tau_{3}} e^{i \omega_{4} \tau^{\prime}} T_{L I, k^{\prime} m^{\prime}}^{n^{\prime} n, a}\left(\omega_{4}\right) .
\end{aligned}
$$


The integral is triple over time $\left(\tau_{1}, \tau_{2}, \tau_{3}\right)$ and in the last stage fourfold over frequency $\left(\omega_{1}\right.$ to $\left.\omega_{4}\right)$. Recognizing representations of $\delta$ distributions in the integrals over $\tau_{i}$ and renaming $\omega=\omega_{1}$, we are left with

$$
\begin{aligned}
\sum_{n^{\prime}} \sum_{k} \sum_{m} \sum_{k^{\prime}} \sum_{m^{\prime}} \int d \omega g_{I I}^{r}(\omega) T_{I L, k m}^{n n^{\prime}, r}(\omega) g_{L L}^{+-}(\omega+k A+m B) \\
\quad \times T_{L I, k^{\prime} m^{\prime}}^{n^{\prime} n, a}\left(\omega+\left(k-k^{\prime}\right) A+\left(m-m^{\prime}\right) B\right) \\
\quad \times e^{-i \omega \tau} e^{i\left[\omega+\left(k-k^{\prime}\right) A+\left(m-m^{\prime}\right) B\right] \tau^{\prime}} .
\end{aligned}
$$

Now setting $\tau=\tau^{\prime}$ and demanding that no phase factor depending on $\tau$ remains, necessitates $k^{\prime}=k$ and $m^{\prime}=m$, thus the result from Eq. (2.16). ac current contributions in a channel do not alter the probabilities for the island charge states in a long-time average and do not have to be counterbalanced by other processes, transfers through other channels or the other junction in order to keep the $P_{n}$ constant. Therefore in analogy to the dc current in Ref. 13 here already the single-channel island charge-change rates are restricted to their nonoscillating parts.
*Electronic address: Ursula.Schroeter@uni-konstanz.de

${ }^{1}$ H. Grabert and M. Devoret, Single Charge Tunneling (Plenum, New York, 1992).

${ }^{2}$ W. Belzig and Y. Nazarov, Phys. Rev. Lett. 87, 197006 (2001).

${ }^{3}$ Y. Avishai, A. Golub, and A. D. Zaikin, Europhys. Lett. 54, 640 (2001).

${ }^{4}$ T. Pohjola, J. König, M. M. Salomaa, J. Schmid, H. Schoeller, and G. Schön, Europhys. Lett. 40, 189 (1997).

${ }^{5}$ J. Heurich, J. C. Cuevas, W. Wenzel, and G. Schön, Phys. Rev. Lett. 88, 256803 (2002).

${ }^{6}$ A. Furusaki and K. A. Matveev, Phys. Rev. Lett. 75, 709 (1995).

${ }^{7}$ I. L. Aleiner, P. W. Brouer, and L. I. Glazman, Phys. Rep. 358, 309 (2002).

${ }^{8}$ K. Flensberg, Phys. Rev. B 48, 11156 (1993).

${ }^{9}$ N. Agrait, A. Levy-Yeyati, and J. M. van Ruitenbeek, Phys. Rep. 377, 81 (2003).

${ }^{10}$ M. Büttiker, Phys. Rev. B 41, 7906 (1990).

${ }^{11}$ E. Scheer, P. Joyez, D. Esteve, C. Urbina, and M. H. Devoret, Phys. Rev. Lett. 78, 3535 (1997).

${ }^{12}$ E. Scheer, N. Agrait, J. C. Cuevas, A. Levy-Yeyati, B. Ludoph, A. Martin-Rodero, G. Rubio-Bollinger, J. M. van Ruitenbeek, and C. Urbina, Nature (London) 394, 154 (1998).

${ }^{13}$ J. C. Cuevas, A. Martin-Rodero, and A. Levy-Yeyati, Phys. Rev. B 54, 7366 (1996).

${ }^{14}$ G. Metadilis and P. Bruno, Phys. Rev. B 72, 235304 (2005).

${ }^{15}$ H. Grabert, G. L. Ingold, M. H. Devoret, D. Esteve, H. Pothier, and C. Urbina, Z. Phys. B: Condens. Matter 84, 143 (1991).
${ }^{16}$ J. C. Cuevas and W. Belzig, Phys. Rev. Lett. 91, 187001 (2003).

${ }^{17}$ E. Scheer and C. Urbina (unpublished).

${ }^{18}$ D. Averin and A. Bardas, Phys. Rev. Lett. 75, 1831 (1995).

${ }^{19}$ M. F. Goffman, R. Cron, A. Levy-Yeyati, P. Joyez, M. H. Devoret, D. Esteve, and C. Urbina, Phys. Rev. Lett. 85, 170 (2000).

${ }^{20}$ P. Hadley, E. Delvigne, E. H. Visscher, S. Lahteenmaki, and J. E. Mooij, Phys. Rev. B 58, 15317 (1998).

${ }^{21}$ M. Tinkham, Physica B 204, 176 (1995).

${ }^{22}$ J. C. Cuevas, Ph.D. thesis, Universidad Autonoma de Madrid, 1999.

${ }^{23}$ C. W. J. Beenakker, Phys. Rev. B 46, 12841 (1992).

${ }^{24}$ R. J. Fitzgerald, S. L. Pohlen, and M. Tinkham, Phys. Rev. B 57, R11073 (1998).

${ }^{25}$ T. A. Fulton, P. L. Gammel, D. J. Bishop, L. N. Dunkleberger, and G. J. Dolan, Phys. Rev. Lett. 63, 1307 (1989).

${ }^{26}$ A. M. van den Brink, A. A. Odintsov, P. A. Bobbert, and G. Schön, Z. Phys. B: Condens. Matter 85, 459 (1991).

${ }^{27}$ T. M. Klapwijk, G. E. Blonder, and M. Tinkham, Physica B 109/ 110, 1657 (1982).

${ }^{28}$ M. T. Tuominen, J. M. Hergenrother, T. S. Tighe, and M. Tinkham, Phys. Rev. Lett. 69, 1997 (1992).

${ }^{29}$ J. G. Lu, J. M. Hergenrother, and M. Tinkham, Phys. Rev. B 57, 120 (1998).

${ }^{30}$ J. M. Hergenrother, M. T. Tuominen, J. G. Lu, D. C. Ralph, and M. Tinkham, Physica B 203, 327 (1994). 